\title{
Alleviation of Salt Stress in Nigella Sativa L. By Gibberellic Acid and Rhizobacteria
}

\author{
Nahed, M. Rashed ${ }^{1}$, Awad. Y. Shala ${ }^{2}$, Mahmoud A. Mahmoud $^{3}$
}

\begin{abstract}
Salinity is one of the extremely serious abiotic stresses for plants, affecting other subsequent consequences such as oxidative stress, which finally leads to cell death. A pot experiment was performed during 2014 / 2015 and 2015/ 2016 at Sakha Agricultural Research Station, to elucidate the alleviation of salinity effects by spraying gibberellic acid (GA $)_{3}$, Azospirillum sp. and Azotobacter sp. Rhizobacteria (PGPR) and the combination between $\mathrm{GA}_{3}+$ (PGPR) and their effects on the vegetative growth, yield characters, chemical composition and fixed oil percentage of black cumin plant. Salinity concentrations were 1000, 2000, 3000 and $4000 \mathrm{ppm}$ sea water diluted compared with fresh water as control, $\mathrm{GA}_{3}$ was used at $100 \mathrm{ppm}$ and PGPR at10\%. Salinity treatments significantly decreased plant height, number of branches, plant dry weight, number of capsules, number of roots per plant, root volume, roots fresh and dry weights, capsules yield, seed yield /plant and 1000 seeds weight compared with control. Salinity also decreased chlorophyll content, fixed oil percentage and relative water content. However, proline content, peroxidase and catalase activities, membrane permeability and total soil soluble salts were increased relative to the control. $\mathrm{GA}_{3}$ or PGPR treatments alleviated the above mentioned undesirable effects of salinity. The increment of enzymes activities and proline accumulation due to $\mathrm{GA}_{3}$ or PGPR treatments are suggested to involve as part of the defense versus salinity on Nigella sativa $L$ plants. To reduce the unfavorable salinity influences, treatment of $\mathrm{GA}_{3}$ at 100 ppm or PGPR at10\% was recommended.
\end{abstract}

Keywords: Salinity, Nigella sativa L., gibberellic acid, Rhizobacteria, Seed yield, Fixed oil.

\section{INTRODUCTION}

Nigella sativa L. (black cumin) is an aromatic and medicinal plant from Ranunculaceae family. This plant is customarily utilized as a flavor and as a characteristic cure in the treatment of a few diseases (Cheikh-Rouhou et al., 2007). It exhibited an extensive pharmacological actions (Bourgou et al., 2008, 2010) which due to its abundance in a few secondary metabolites including seed volatile oil (Bourgou et al., 2010), seed fixed oil which, contain linoleic acid (40.3-58.9\%), oleic (18.7$28.1 \%)$, palmitic $(10.1-12.5 \%)$ and stearic $(2.6-3.1 \%)$ acids (Ramadan, 2007 and Matthaus and Ozcan, 2011) and phenolic compounds in the shoots and the roots (Bourgou et al., 2008). The previous both organs are chiefly rich in vanillic acid. This plant has been expanded as a usual remedy for illnesses for example, asthma, irritation, diabetes, tumor, gastrointestinal unsettling influences, hypertension, and gynecological disorders for over several years (Ramadan, 2007).

Egyptian economy depends on a great degree on agriculture. The rapidly rising population and variations in the way of life require judicious advancement in agricultural production. Thus, the prominent goal of the Egyptian policy is to rise the land production through better land usage, improvement of agricultural techniques and bring new land areas to cultivation. The Egyptian budget of the Nile freshwater is low and its quantity approximately 55.5 milliard $\mathrm{m}^{3}$. Looking at the upcoming of stressing water demands, it is quite obvious, that a very careful use of accessible water sources and expansion of new resources such as drainage, well, sewage and sea water should be contemplated. Irrigation by saline water may decrease crops yield. Although, using sea water in irrigation may save the fresh water resources for the other usages but, what about the effect of using sea water in agriculture?

Plants grown in farming systems are subjected to numerous abiotic and biotic stresses which reduce their quality and revenue potential. Salinity remains the basic reason which, decreasing plant growth then productivity worldwide. It influences around 7\% of the world's whole land area (Flowers et al., 1997 and Zhu, 2002). Salinity stress influences growth besides metabolic activities of plant species (Baghalian et al., 2008 and Oueslati et al., 2010). Upon observing environmental stresses plants enact a range of resistance mechanisms which might also be made artificially or boosted by

${ }^{1}$ Vegetable and Floriculture Dept., Fac. of Agric. Damietta Univ.,

Damietta, Egypt.

E-mail address: rashed_nahed@du.edu.eg

${ }^{2}$ Medicinal and Aromatic Plants Research Department, Horticulture

Research Institute. Agricultural Research Center, Giza, Egypt.

E-mail address awad.shala@yahoo.com

${ }^{3}$ Water Requirements and Field Irrigation Department, Soils,

Water and Environment Research Institute. Agricultural Research

Center, Giza, Egypt.

E-mail address mahmoud_abdalla96@yahoo.com

Received October 16,2017, Accepted November29, 2017 
using specific chemicals (Rajasekaran and Blake, 1999). These days consideration has been directed to practical and environment-friendly substitutes for example, biological ways to improve and encourage plant growth. Beneficial bacteria, particularly in plants rhizosphere have been examined and established to have growthpromoting activities. The impact of PGPR on the alleviation of salinity has been stated (Weyens et al., 2009 and Yang et al., 2009)

Also, other attempts have been made to alleviate deleterious effects of salinity; different types of phytohormons are being used. Of these, gibberellic acid is an essential phytohormone able to impart stress tolerance involving salinity, in several plants (Hoque and Haque, 2002). The $\mathrm{GA}_{3}$ has significantly impact the procedures of seed germination, leaf extension, stem stretching, bloom and trichome origination, and fruit development (Yamaguchi, 2008). Through their impact on photosynthetic enzymes, $\mathrm{GA}_{3}$ is identified to increase the photosynthetic efficacy of plants, leaf area index, light capture, the efficiency of nutrients and assume an essential part in regulating various processes through plant development (Khan, et al. 2010). $\mathrm{GA}_{3}$ has been accounted to reduce the undesirable consequences of salinity plant water relationships in addition water use efficiency (Yamaguchi, 2008). The effect of $\mathrm{GA}_{3}$ on salinity mitigation has been previously reported (Maggio et al., 2010) on tomato plants, (Saeidi-Sar et al., 2013) on (Phaseolus vulgaris L. cv. Naz) otherwise ( Khan, et al. 2010) on (Linum usitatissimum L.).

Under stress, plants established compound mechanisms to combat against these oxidative stresses via the synchronous activity of various antioxidants. Of these, superoxide dismutase (SOD) which changes superoxide to $\mathrm{H}_{2} \mathrm{O}_{2}$, peroxidase (POD) which changes $\mathrm{H}_{2} \mathrm{O}_{2}$ to water and catalase (CAT) eliminates $\mathrm{H}_{2} \mathrm{O}_{2}$. Also, plants adjust osmotic stress by gathering some compatible solutes for example, proline, glycinebetaine, polyols and trehalose (Ghoulam et al., 2002 and Sakamoto and Murata, 2002). Proline plays a key role in keeping plants from osmotic stress. Thus, antioxidants besides compatible solutes could supply approach to boost plants salt tolerance. Concerning Nigella sativa L. the effect of salinity on leaves fatty

Table A. Some physical and chemical soil properties of the used medium as mean values of the two experimental growth season

\begin{tabular}{|c|c|c|c|c|c|c|c|c|}
\hline $\begin{array}{c}\text { Field } \\
\text { capacity } \\
(\%)\end{array}$ & $\begin{array}{c}\text { Wilting point } \\
(\%)\end{array}$ & $\begin{array}{l}\text { Bulk density } \\
\quad\left(\mathrm{mg} \mathrm{m}^{-3}\right)\end{array}$ & $\begin{array}{c}\text { Total } \\
\text { porosity } \\
(\%)\end{array}$ & $\begin{array}{c}\text { Sand } \\
(\%)\end{array}$ & $\begin{array}{l}\text { Silt } \\
(\%)\end{array}$ & $\begin{array}{l}\text { Clay } \\
(\%)\end{array}$ & $\begin{array}{c}\text { Texture } \\
\text { class }\end{array}$ & pH \\
\hline 44.62 & 22.83 & 1.16 & 56.23 & 19.16 & 26.52 & 54.32 & Clayey & 8.21 \\
\hline $\mathrm{EC}_{\mathrm{e}}$ & \multicolumn{4}{|c|}{ Inions concentration meq/L } & \multicolumn{4}{|c|}{ Cations concentration $\mathrm{meq} / \mathrm{L}$} \\
\hline$\left(\mathrm{dS} \mathrm{m} \mathrm{m}^{-1}\right)$ & $\mathrm{CO}_{3}{ }^{--}$ & $\mathrm{HCO}_{3}^{-}$ & $\mathrm{Cl}^{-}$ & $\mathrm{SO}_{4}^{--}$ & $\mathrm{Ca}^{++}$ & $\mathrm{Mg}^{++}$ & $\mathrm{Na}^{+}$ & $\mathrm{K}^{+}$ \\
\hline 3.73 & --- & 4.60 & 16.48 & 17.18 & 7.35 & 8.11 & 22.47 & 0.33 \\
\hline
\end{tabular}

acid content has been studied by (Bourgou et al., 2012) . However, no data have been collected regarding seeds fatty acid (fixed oil) content under saline sea water. Nevertheless, to the best of our insight, no published literature exists about sea water salinity effects on fatty acid content. Therefore, this study attempted to investigate for the first time the effect of PGPR and $\mathrm{GA}_{3}$ on growth attributes and biochemical characters under different concentrations of saline sea water, in order to use of Nigella sativa L.as an economic substitute for field crops and to save freshwater.

\section{MATERIALS AND METHODS}

\section{Field site description}

A pot experiment was carried out at Sakha Agricultural Research Station ( $31^{\circ} 07^{\prime} \mathrm{N}$ Latitude, $30^{\circ}$ 05' E Longitude), Kafr El-Sheikh Governorate, North Nile Delta of Egypt during 2014 / 2015 and 2015/ 2016 growing seasons to study the impact of irrigation with sea-fresh mixed water, of increased salinity levels. The experiment was performed using complete randomized blocks design with four replications. Plastic pots with a top diameter of $30 \mathrm{~cm}$ and a depth of $18 \mathrm{~cm}$ were filled with 5-kilogram clayey soil. Physical and chemical soil properties of the experimental site was showed in Table (A). Black cumin seeds were acquired from Medicinal and Aromatic Plants Research Department, Horticulture Research Institute, Agricultural Research Center, Egypt. Ten seeds were sown on December $1^{\text {st }}, 2014$ and 2015 in every pot and after six weeks then they were thinned to five healthy seedlings per pot.

\section{Experimental treatments:}

\section{Salinity treatments}

The plants irrigated with freshwater $289 \mathrm{ppm}$ $(0.45 \mathrm{ds} / \mathrm{m})$ from sowing until 60 days, then salinity treatments were applied after the seedlings started their growth and development until harvesting on May $1^{\text {st }}$ for both seasons. The plants were given water requirements plus $20 \%$ as leaching requirements for all treatments through all seasons until harvest. The salinity levels were obtained by addition of appropriate quantity of sea water to freshwater and were adjusted through a portable Ec meter instrument. 
The treatments were as the following

$$
\begin{aligned}
& \text { 1-Control }(0.45 \mathrm{ds} / \mathrm{m}) . \quad 2-1000 \mathrm{ppm} \\
& \text { (1.562ds/m). 3- } 2000 \mathrm{ppm}(3.125 \mathrm{ds} / \mathrm{m}) \text {. 4- } \\
& 3000 \mathrm{ppm}(4.69 \mathrm{ds} / \mathrm{m}) \text {. } \\
& \text { 5- } 4000 \mathrm{ppm}(6.25 \mathrm{ds} / \mathrm{m}) \text {. }
\end{aligned}
$$

\section{Spraying treatments}

\section{Gibberellic acid $\left(\mathbf{G A}_{3}\right)$}

Gibberellin $100 \mathrm{ppm}$ was sprayed twice in the morning during the vegetative stage, the first one was on February $15^{\text {th }}$ and $17^{\text {th }}$ in both seasons, respectively. So, this means that after one week from the beginning of the saline irrigation treatments, while the second spray was on March $4^{\text {th }}$ and $6^{\text {th }}$ in both seasons, respectively

\section{Rhizobacteria inoculants (PGPR)}

Selected strains of Rhizobacteria (PGPR) were Azospirillum sp. and Azotobacter sp. both cultures were kindly supplemented by Microbiology Department, Sakha Agricultural Research Station in modified tryptone yeast extract and glucose (TYG) ( Jensen, 1951 and Bashan et al., 2002) both media with cell density 4 x $10^{11}$ and $9 \times 10^{9}$ for Azospirillum sp and Azotobacter sp respectively. Rhizobacteria were sprayed twice as mentioned in $\mathrm{GA}_{3}$ at a concentration of $10 \%$.

\section{Gibberellic acid $\left(\mathbf{G A}_{3}\right)$ plus Rhizobacteria inoculants(PGPR)}

$\mathrm{GA}_{3}$ and PGPR were dissolved separately in distilled water as a solution and added as a foliar application using a sprayer and conical bowl which was putted on top of the pots to concentrate spraying on treated plants and prevents spraying other plants. Gibberellic acid was sprayed on the early morning then after two hours, the microbial (PGPR) was sprayed twice in the meantime of the previous treatments $\left(\mathrm{GA}_{3}\right)$.

\section{Freshwater}

Tap water $(0.45 \mathrm{ds} / \mathrm{m})$ was sprayed twice in the meantime of the previous treatments as a control.

\section{Harvest time}

The plants were gathered at full maturity stage on May $1^{\text {st }}$ in the two seasons.

\section{Collected data}

\section{Growth and yield characters}

\section{Vegetative growth and yield characters}

The height of plants was measured in centimeters of the main stem from ground level to the plant top by measuring tape. The number of branches/plant was determined by counting the number of reproductive branches that appeared within growing season from each plant. Plant dry weight $(\mathrm{g})$ : The plants mentioned above were cut, then placed in an envelope and dried naturally in the lap. Capsules were picked randomly from plants, put in a small envelope bag and weighted $(\mathrm{g})$, then determined average capsules number and weight per plant. Seeds yield /plant (g) capsules were picked randomly from plants, shelling the seeds from capsules after physically, drying in the lap their seeds were added to their respective seeds of the capsules in the small bags and weighted, and dry weight of 1000 seeds (g) was estimated by counting 1000-seeds randomly from each pot five times and weighted using a sensitive balance for both seasons.

\section{Root characters}

Roots length, roots number, fresh and dry weight and root volume. Plant samples of each plant were taken at harvesting time, washed with distilled water, then spread roots and shoots. Main roots were counted as a number and the length of the main root was measured using scale ruler. Root weighted as fresh using sensitive balance, oven dried at $70{ }^{\circ} \mathrm{C}$ even weight stability, dry weight was recorded, ground and kept for analyses. Root volume was determined by water relocation methods, the measuring was prepared in a unique container with an overflow spout. This container is loaded with water until it floods from the spout. Then fresh washed roots which have been carefully dried with a soft cloth are immersed and the flood water volume is measured in a graduated cylinder (Bohm, 2012).

\section{Biochemical characters \\ Chlorophyll Content}

Randomly samples of new leaves April1 ${ }^{\text {st }}$ were grabbed from the central part of the stem for chlorophyll determination. Chlorophyll a and b mg/g F.W were determined by the method defined by (Moran, 1982) by using spectrophotometer (Pharmacia, LKB-Novaspec II)

\section{Relative Water Content of Leaves (RWC)}

The relative water content of leaves (RWC) was estimated by the method of (Whetherley, 1950). Leaf material was balanced $(0.5 \mathrm{~g})$ to establish fresh weight (FW) and located in double-distilled water for $4 \mathrm{~h}$, subsequently this time turgid weight (TW) was recorded. Subsequently, the samples were saved in a hot air oven at $65{ }^{\circ} \mathrm{C}$ for $48 \mathrm{~h}$ and their dry weights (DW) were recorded. RWC was calculated as:

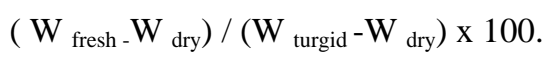

\section{Membrane permeability (Mb)}

Membrane permeability of the excised leaves was measured at the completion of the experiment (Yan et al., 1996) fresh portion from the center of leaves was 
balanced into a glass beaker comprising reverse osmosis water. The beakers were dipped at $30 \pm 1{ }^{\circ} \mathrm{C}$ for $3 \mathrm{~h}$, and subsequently the conductivity of the solution was calculated with a conductivity meter. The conductivity was determined again next boiling the samples for 2 min. once the solution was air-conditioned to room temperature. The percentage of electrolyte leakage was considered by implementation of the formula, EC \% = $(\mathrm{C} 1 / \mathrm{C} 2) \mathrm{X} 100$, since $\mathrm{C} 1$ and $\mathrm{C} 2$ are the electrolyte conductivities evaluated before and after boiling, respectively.

\section{Fixed oil content}

The air dried seeds balanced (50 g) were powdered mechanically and extracted with light petroleum ether $\left(60-80^{\circ} \mathrm{C}\right)$ for $4 \mathrm{~h}$ in a Soxhlet apparatus. Removal of the solvent was done under reduced pressure gave the fixed oils (Horwitz et al., 1970).

\section{Proline}

The free proline content was determined according to (Bates et al., 1973). Frozen leaf tissue $(0.5 \mathrm{~g})$ was homogenized with $10 \mathrm{ml}$ of $3 \%$ sulfosalicylic acid at 4 ${ }^{\circ} \mathrm{C}$. Then, the acquired extract was clarified with Whatman No. 2. A mixture of $2 \mathrm{ml}$ of the filtrate, $2 \mathrm{~mL}$ from acid-ninhydrin, and $2 \mathrm{~mL}$ of glacial acetic acid was mixed inside a test tube and incubated at $100{ }^{\circ} \mathrm{C}$ for $1 \mathrm{~h}$. The reaction was done on the ice, and the reaction combination was then separated with $4 \mathrm{~mL}$ of toluene. The chromophore-containing toluene was removed from the hydrated stage. The absorbance at $520 \mathrm{~nm}$ was spectrophotometrically defined with toluene as the blank. The proline concentration was calculated established on a standard curve and was communicated as $\mu \mathrm{mol} \mathrm{g}^{-1} \mathrm{~F}$.W.

\section{Antioxidant Enzyme Activity}

To obtain the enzyme extract for antioxidant enzymes determination, the method formerly described by (Hassan and Mahfouz, 2012) was used. The subsequent supernatant was consumed as an enzyme extract to determine peroxidase (POX) and catalase (CAT) activities. Soluble protein contents of the enzyme extract were assessed according to (Bradford, 1976).

\section{Peroxidase activity}

was tested according to (Shannon et al., 1966). Sodium acetate buffer $(0.1 \mathrm{M})$ and $0.5 \%$ guaiacol were added to the enzyme extract. The reaction was commenced with $0.1 \% \mathrm{H}_{2} \mathrm{O}_{2}$. The rate of variation in absorbance was spectrophotometrically measured at 470 $\mathrm{nm}$ and quantity of enzyme activity was communicated

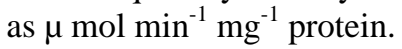

\section{Catalase activity}

was spectrophotometrically evaluated by (Claiborne, 1985) following the disappearance of $\mathrm{H}_{2} \mathrm{O}_{2}$ at $240 \mathrm{~nm}$. The amount of enzyme activity was stated as $\mu \mathrm{mol} \mathrm{min}{ }^{-}$ ${ }^{1} \mathrm{mg}^{-1}$ protein.

\section{Total soluble salts}

At the completion of the experiments soil samples were taken from every pot and chemically analyzed, total soluble salts were measured by electrical conductivity (EC) apparatus in the saturated soil paste extract (Page et al., 1982).

\section{Statistical analysis}

Data for each season were evaluated by the method defined by (Steel et al., 1980) and differences between the means were investigated by Duncan's Multiple Range Test (Snedecor and Cochran, 1980) using COSTAT computer program.

\section{RESULTS}

\section{Growth and yield characteristics}

\section{Vegetative growth characteristics}

Salinity treatments adversely affected on plant height, branches number, plant dry weight and capsules number compared to control in both seasons (Table 1). Plants received freshwater (without salinity) gave the highest significant mean values for plant height, branches number, plant dry weight and capsules number for both seasons. Expanding salinity levels gradually decreased all previously mentioned characters. Generally, the overall mean values for this characters can be descended in order 1000ppm >2000ppm $>3000 \mathrm{ppm}>4000 \mathrm{ppm}$ in most cases for the two seasons. The decline in plant height, branches number, plant dry weight and capsules number by salinity was alleviated when $\mathrm{GA}_{3}$ or PGPR or $\mathrm{GA}_{3}+$ PGPR were applied. Application of $\mathrm{GA}_{3}$ positively improved plant height better than PGPR or $\mathrm{GA}_{3}+$ PGPR. Moreover, applying PGPR enhanced the branches number which led to increasing plant dry weight and capsules number for both seasons. The interaction among different saline water and exogenous $\mathrm{GA}_{3}$ application recorded the highest plants under different saline water concentrations. Among all treatments applied, the tallest black cumin plants were recorded by $\mathrm{GA}_{3}$ with 1000 ppm or control treatment without significant variations among them in the first season and $\mathrm{GA}_{3}$ with 2000 ppm in the second season. Applied PGPR with salinity at $2000 \mathrm{ppm}$ in the first season and PGPR with salinity at $1000 \mathrm{ppm}$ in the subsequent season recorded the highest branches number per Nigella sativa L. plant. Additionally, using PGPR with 1000 ppm saline water achieved the heaviest dry weight plus the highest capsules number for both seasons. 
Root growth characteristics

Table 1. Effect of saline irrigation water levels and sprayi number, plant dry weight and capsules number of Nigella sativa L. plants

\begin{tabular}{|c|c|c|c|c|c|c|c|c|c|c|}
\hline \multirow{3}{*}{$\begin{array}{l}\text { Seasons } \\
\text { Spraying } \\
\text { Salinity }\end{array}$} & \multicolumn{5}{|c|}{$1^{\text {st }}$ Season 2015} & \multicolumn{5}{|c|}{$2^{\text {nd }}$ Season 2016} \\
\hline & $\begin{array}{l}\text { Fresh } \\
\text { water }\end{array}$ & $\mathbf{G A}_{3}$ & PGPR & $\begin{array}{l}\text { GA }_{3}^{+} \\
\text {PGPR }\end{array}$ & Mean & $\begin{array}{l}\text { Fresh } \\
\text { water }\end{array}$ & $\mathbf{G A}_{3}$ & PGPR & $\begin{array}{l}\text { GA }^{+} \\
\text {PGPR }\end{array}$ & Mean \\
\hline & \multicolumn{5}{|c|}{ Plant height $(\mathrm{cm})$} & \multicolumn{5}{|c|}{ Plant height $(\mathrm{cm})$} \\
\hline Control & $44.67 f-j$ & $67.00 \mathrm{a}$ & $49.33 d-f$ & $56.67 \mathrm{bc}$ & $54.41 \mathrm{a}$ & $37.67 \mathrm{fg}$ & $50.00 \mathrm{ab}$ & $43.00 \mathrm{de}$ & $48.33 b$ & $44.75 \mathrm{a}$ \\
\hline 1000ppm & $42.67 \mathrm{~h}-\mathrm{j}$ & $65.33 \mathrm{a}$ & $46.67 \mathrm{e}-\mathrm{i}$ & $52.67 \mathrm{~cd}$ & $51.83 b$ & $35.67 \mathrm{~g}$ & $49.33 b$ & $43.33 d$ & $47.67 \mathrm{bc}$ & $44.00 \mathrm{ab}$ \\
\hline 2000ppm & 48.00de-g & $58.67 \mathrm{~b}$ & $42.00 \mathrm{~h}-\mathrm{j}$ & $51.33 \mathrm{de}$ & $50.00 \mathrm{bc}$ & $44.33 d$ & $52.67 \mathrm{a}$ & $36.00 \mathrm{~g}$ & $44.33 d$ & $44.33 \mathrm{ab}$ \\
\hline 3000ppm & $40.67 \mathrm{j}$ & $56.67 \mathrm{bc}$ & $47.33 \mathrm{e}-\mathrm{h}$ & $48.67 d-g$ & $48.33 \mathrm{~cd}$ & $36.33 \mathrm{~g}$ & $50.67 \mathrm{ab}$ & $40.00 \mathrm{ef}$ & $44.33 d$ & $42.83 b$ \\
\hline 4000ppm & $40.67 \mathrm{j}$ & $51.67 \mathrm{~cd} \epsilon$ & $43.67 \mathrm{~g}-\mathrm{j}$ & $51.67 \mathrm{c}-\mathrm{e}$ & $46.92 d$ & $36.00 \mathrm{~g}$ & $44.67 \mathrm{~cd}$ & $38.33 \mathrm{fg}$ & 43.00de & $40.50 \mathrm{c}$ \\
\hline \multirow[t]{2}{*}{ Mean } & $43.33 d$ & $59.87 \mathrm{a}$ & $45.80 \mathrm{c}$ & $52.2 \mathrm{~b}$ & & $38.00 \mathrm{~d}$ & $49.47 \mathrm{a}$ & $40.13 c$ & $45.53 b$ & \\
\hline & \multicolumn{5}{|c|}{ Branches number } & \multicolumn{5}{|c|}{ Branches number } \\
\hline Control & $5.00 \mathrm{~b}$ & $6.00 \mathrm{a}$ & $6.00 \mathrm{a}$ & $5.00 \mathrm{~b}$ & $5.75 \mathrm{a}$ & $7.00 \mathrm{ab}$ & $6.67 \mathrm{a}-\mathrm{c}$ & $7.00 \mathrm{ab}$ & $5.33 \mathrm{ef}$ & $6.5 \mathrm{a}$ \\
\hline 1000ppm & $6.00 \mathrm{a}$ & $4.67 \mathrm{~cd}$ & $6.00 \mathrm{a}$ & $4.00 \mathrm{ef}$ & $4.92 b$ & $7.00 \mathrm{ab}$ & $6.33 b-d$ & $7.33 \mathrm{a}$ & $5.00 \mathrm{fg}$ & $6.42 \mathrm{ab}$ \\
\hline 2000ppm & $5.00 \mathrm{~b}$ & $5.00 \mathrm{~b}$ & $6.00 \mathrm{a}$ & $3.67 f$ & $4.92 b$ & $6.67 \mathrm{a}-\mathrm{c}$ & $5.67 \mathrm{~d}-\mathrm{f}$ & $7.00 \mathrm{ab}$ & $5.33 \mathrm{ef}$ & $6.17 b c$ \\
\hline 3000ppm & $6.00 \mathrm{a}$ & $4.33 \mathrm{de}$ & $5.33 b$ & $4.00 \mathrm{ef}$ & $4.92 b$ & $6.00 \mathrm{cde}$ & $6.33 b-d$ & $7.00 \mathrm{ab}$ & $4.33 \mathrm{~g}$ & $5.92 \mathrm{c}$ \\
\hline $4000 \mathrm{ppm}$ & $4.00 \mathrm{ef}$ & $6.00 \mathrm{a}$ & $6.00 \mathrm{a}$ & $4.00 \mathrm{ef}$ & $5.00 \mathrm{~b}$ & $5.00 \mathrm{fg}$ & $7.00 \mathrm{ab}$ & $7.00 \mathrm{ab}$ & $5.00 \mathrm{fg}$ & $6.00 \mathrm{c}$ \\
\hline \multirow[t]{2}{*}{ Mean } & $5.20 \mathrm{~b}$ & $5.20 \mathrm{~b}$ & $5.87 \mathrm{a}$ & $4.13 c$ & & $6.33 b$ & $6.40 \mathrm{~b}$ & $7.07 \mathrm{a}$ & $5.00 \mathrm{c}$ & \\
\hline & \multicolumn{5}{|c|}{ Plant dry weight (g /plant) } & \multicolumn{5}{|c|}{ Plant dry weight (g /plant) } \\
\hline Control & $6.49 \mathrm{~cd}$ & $7.28 \mathrm{~b}$ & $6.89 b c$ & $6.45 \mathrm{~cd}$ & $6.78 \mathrm{a}$ & $5.61 \mathrm{a}$ & $5.89 \mathrm{a}$ & $5.63 \mathrm{a}$ & $5.75 \mathrm{a}$ & $5.72 \mathrm{a}$ \\
\hline $1000 \mathrm{ppm}$ & $6.91 \mathrm{bc}$ & $4.94 \mathrm{fg}$ & $8.11 \mathrm{a}$ & $4.28 \mathrm{hi}$ & $6.06 \mathrm{~b}$ & $5.28 \mathrm{a}$ & $3.51 \mathrm{a}$ & $6.15 \mathrm{a}$ & $3.65 \mathrm{a}$ & $4.64 b$ \\
\hline 2000ppm & $5.21 \mathrm{ef}$ & $4.82 \mathrm{fgh}$ & $6.26 \mathrm{~d}$ & $5.55 \mathrm{e}$ & $5.46 \mathrm{c}$ & $4.63 \mathrm{a}$ & $4.07 \mathrm{a}$ & & $4.60 \mathrm{a}$ & $4.68 b$ \\
\hline $3000 \mathrm{ppm}$ & $4.52 \mathrm{gh}$ & $3.81 \mathrm{i}$ & $5.66 \mathrm{e}$ & 4.70fgh & $4.67 \mathrm{~d}$ & $3.82 \mathrm{a}$ & $3.10 \mathrm{a}$ & $4.87 \mathrm{a}$ & $3.61 \mathrm{a}$ & $3.85 \mathrm{c}$ \\
\hline 4000ppm & $3.89 \mathrm{i}$ & $3.70 \mathrm{i}$ & $7.42 b$ & $4.71 \mathrm{fgh}$ & $4.93 \mathrm{~d}$ & $3.30 \mathrm{a}$ & $3.12 \mathrm{a}$ & $6.39 \mathrm{a}$ & $3.49 \mathrm{a}$ & $4.08 \mathrm{c}$ \\
\hline \multirow[t]{2}{*}{ Mean } & $5.41 \mathrm{~b}$ & $4.91 \mathrm{~d}$ & $6.87 \mathrm{a}$ & $5.14 \mathrm{c}$ & & $4.53 b$ & $3.94 d$ & $5.69 \mathrm{a}$ & $4.22 \mathrm{c}$ & \\
\hline & & \multicolumn{4}{|c|}{ Capsules number } & \multicolumn{4}{|c|}{ Capsules number } & \\
\hline Control & $12.67 \mathrm{bc}$ & $13.33 b$ & $17.67 \mathrm{a}$ & $12.00 \mathrm{bc}$ & $13.92 \mathrm{a}$ & $11.00 \mathrm{c}$ & $10.00 \mathrm{~d}-1$ & $13.33 \mathrm{a}$ & $8.33 \mathrm{~h}$ & $10.67 \mathrm{a}$ \\
\hline 1000ppm & $13.00 \mathrm{bc}$ & $12.00 \mathrm{bc}$ & $17.00 \mathrm{a}$ & $12.00 \mathrm{bc}$ & $13.50 \mathrm{a}$ & $10.00 \mathrm{~d}-\mathrm{f}$ & $10.33 c-6$ & $13.33 \mathrm{a}$ & $9.33 \mathrm{fg}$ & $10.67 \mathrm{a}$ \\
\hline 2000ppm & $12.67 \mathrm{bc}$ & $12.00 \mathrm{bc}$ & $16.33 \mathrm{a}$ & $11.00 \mathrm{~cd}$ & $13.00 \mathrm{ab}$ & $11.00 \mathrm{c}$ & $11.00 \mathrm{c}$ & $10.67 \mathrm{~cd}$ & $6.33 \mathrm{i}^{\circ}$ & $9.75 \mathrm{c}$ \\
\hline $3000 \mathrm{ppm}$ & $9.33 \mathrm{de}$ & $13.33 b$ & $13.67 b$ & $13.00 \mathrm{bc}$ & $12.33 \mathrm{bc}$ & $9.67 \mathrm{e}-\mathrm{g}$ & $9.00 \mathrm{~g}$ & $12.67 \mathrm{~b}$ & $9.00 \mathrm{~g}$ & $10.17 b$ \\
\hline $4000 \mathrm{ppm}$ & $12.67 \mathrm{bc}$ & $12.67 \mathrm{bc}$ & $13.33 b$ & $8.67 \mathrm{e}$ & $11.83 \mathrm{c}$ & $6.00 \mathrm{i}$ & $10.67 \mathrm{~cd}$ & $11.00 \mathrm{c}$ & $6.33 \mathrm{i}$ & $8.50 \mathrm{~d}$ \\
\hline Mean & $12.07 \mathrm{bc}$ & $12.67 \mathrm{~b}$ & $15.60 \mathrm{a}$ & $11.33 \mathrm{c}$ & & $9.53 c$ & $10.20 \mathrm{~b}$ & $12.20 \mathrm{a}$ & $7.87 \mathrm{~d}$ & \\
\hline
\end{tabular}

Means designed by the same letter at each cell are not significantly different at the 5\% level according to Duncan's multiple range tests.

(Table 2). While, salinity treatments significantly decreased (root length, root number, root volume, root fresh and dry weight). Using $\mathrm{GA}_{3}$ or PGPR or $\mathrm{GA}_{3}+$ PGPR significantly increased them when applied or minimized the reduction occurred by salinity. The most efficient treatment in this concern was $\mathrm{GA}_{3}$ which promoted root length, root volume, root fresh and dry weight for both seasons. Otherwise, $\mathrm{GA}_{3}+$ PGPR significantly boosted roots number. Moreover, a combination between $\mathrm{GA}_{3}$ with salinity mostly caused a noticeable root length increment and root volume in the two seasons. Also, the inhibitory impact of salinity stress was completely ameliorated generally at low salinity level $(1000 \mathrm{ppm})$ with $\mathrm{GA}_{3}$ especially in root fresh and dry weight for both seasons.

Yield characteristics
All root characters (root length, roots number, root volume, root fresh and dry weight) were significantly influenced by salinity and salinity alleviators treatments
From Table 3 and Fig 1, a gradual reduction in capsules dry weight, seeds yield/plant and 1000 seeds weight with increasing of salinity levels could be noticed; the least values in this concern were obtained from the elevated level (4000ppm) for all parameters in both seasons. Otherwise, salinity treatments caused more reduction in seeds yield/plant reached 67.39 and $80.37 \%$ at $4000 \mathrm{ppm}$ for both seasons, respectively. Although $\mathrm{GA}_{3}$, PGPR and $\mathrm{GA}_{3}+$ PGPR alleviated the adverse salinity influences on capsules dry weight, seeds yield/plant and 1000 seeds weight. Applying PGPR enhanced capsules dry weight and seeds yield/plant. Moreover,

increasing of seeds yield/plant by applying PGPR reached to 46.88 and $60.40 \%$ in both seasons, respectively while, using $\mathrm{GA}_{3}$ increased 1000 seeds weight. The highest capsules dry weight was obtained 
from plants treated with $\mathrm{GA}_{3}+$ PGPR without salinity yield/plant and 1000 seeds weight was also noticed for both growth seasons. A promotion effect on seeds

Table 2. Effect of saline irrigation water levels and treatment with $\mathrm{GA}_{3}$ or PGPR on root length, root number, root volume, root fresh and dry weights of $N$. sativa $L$. plants

\begin{tabular}{|c|c|c|c|c|c|c|c|c|c|c|}
\hline \multirow{3}{*}{$\begin{array}{l}\text { Seasons } \\
\text { Spraying } \\
\text { Salinity }\end{array}$} & \multicolumn{5}{|c|}{$1^{\text {st }}$ Season 2015} & \multicolumn{5}{|c|}{$2^{\text {nd }}$ Season 2016} \\
\hline & $\begin{array}{l}\text { Fresh } \\
\text { water }\end{array}$ & $\mathbf{G A}_{3}$ & PGPR & $\begin{array}{l}\mathbf{G A}_{3}+ \\
\text { PGPR }\end{array}$ & Mean & Fresh wate & $\mathbf{G A} \mathbf{A}_{3}$ & PGPR & $\begin{array}{l}\mathbf{G A}_{3}+ \\
\text { PGPR }\end{array}$ & Mean \\
\hline & \multicolumn{5}{|c|}{ Root length $(\mathrm{cm})$} & \multicolumn{5}{|c|}{ Root length $(\mathrm{cm})$} \\
\hline Control & $13.00 \mathrm{e}$ & $17.00 \mathrm{a}$ & $14.00 \mathrm{~d}$ & $14.00 \mathrm{~d}$ & $14.50 \mathrm{a}$ & $11.00 \mathrm{e}$ & $15.00 \mathrm{a}$ & $13.00 \mathrm{c}$ & $12.00 \mathrm{~d}$ & $12.75 \mathrm{a}$ \\
\hline $1000 \mathrm{ppm}$ & $14.00 \mathrm{~d}$ & $17.00 \mathrm{a}$ & $14.00 \mathrm{~d}$ & $13.00 \mathrm{e}$ & $14.50 \mathrm{a}$ & $13.00 \mathrm{c}$ & $13.00 \mathrm{c}$ & $14.00 \mathrm{~b}$ & $11.00 \mathrm{e}$ & $12.75 \mathrm{a}$ \\
\hline 2000ppm & $16.00 \mathrm{~b}$ & $12.00 \mathrm{f}$ & $15.00 \mathrm{c}$ & $12.33 \mathrm{f}$ & $13.83 b$ & $11.00 \mathrm{e}$ & $11.00 \mathrm{e}$ & $14.00 \mathrm{~b}$ & $12.00 \mathrm{~d}$ & $12.00 \mathrm{~b}$ \\
\hline $3000 p p m$ & $12.67 \mathrm{e}$ & $14.00 \mathrm{~d}$ & $13.00 \mathrm{~d}$ & $13.00 \mathrm{e}$ & $13.42 b$ & $13.00 \mathrm{c}$ & $12.00 \mathrm{~d}$ & $11.00 \mathrm{e}$ & $10.67 \mathrm{e}$ & $11.67 \mathrm{c}$ \\
\hline 4000ppm & $13.00 \mathrm{e}$ & $12.00 \mathrm{f}$ & $13.00 \mathrm{e}$ & $13.00 \mathrm{e}$ & $12.75 \mathrm{c}$ & $12.00 \mathrm{~d}$ & $11.70 \mathrm{~d}$ & $10.00 \mathrm{f}$ & $11.00 \mathrm{e}$ & $11.18 \mathrm{~d}$ \\
\hline Mean & $13.73 b$ & $14.40 \mathrm{a}$ & $13.80 \mathrm{~b}$ & $13.07 \mathrm{c}$ & & $12.00 \mathrm{~b}$ & $12.54 \mathrm{a}$ & $12.40 \mathrm{a}$ & $11.33 \mathrm{c}$ & \\
\hline & \multicolumn{5}{|c|}{ Root Number } & \multicolumn{5}{|c|}{ Root Number } \\
\hline Control & $5.00 \mathrm{~d}$ & $6.00 \mathrm{c}$ & $5.00 \mathrm{~d}$ & $8.00 \mathrm{a}$ & $6.00 \mathrm{a}$ & $6.00 \mathrm{~b}$ & $6.00 \mathrm{~b}$ & $5.00 \mathrm{c}$ & $7.00 \mathrm{a}$ & $6.00 \mathrm{a}$ \\
\hline 1000ppm & $5.00 \mathrm{~d}$ & $6.00 \mathrm{c}$ & $5.00 \mathrm{~d}$ & $7.00 \mathrm{~b}$ & $5.75 b$ & $6.00 \mathrm{~b}$ & $5.00 \mathrm{c}$ & $5.00 \mathrm{c}$ & $5.00 \mathrm{c}$ & $5.25 b$ \\
\hline 2000ppm & $6.00 \mathrm{c}$ & $6.00 \mathrm{c}$ & $5.00 \mathrm{~d}$ & $6.00 \mathrm{c}$ & $5.75 b$ & $6.00 \mathrm{~b}$ & $5.00 \mathrm{c}$ & $5.00 \mathrm{c}$ & $5.00 \mathrm{c}$ & $5.25 b$ \\
\hline $3000 \mathrm{ppm}$ & $5.00 \mathrm{~d}$ & $6.00 \mathrm{c}$ & $6.00 \mathrm{c}$ & $6.00 \mathrm{c}$ & $5.75 b$ & $4.00 \mathrm{~d}$ & $5.00 \mathrm{c}$ & $5.00 \mathrm{c}$ & $5.00 \mathrm{c}$ & $4.75 c$ \\
\hline 4000ppm & $6.00 \mathrm{c}$ & $5.00 \mathrm{~d}$ & $5.00 \mathrm{~d}$ & $5.00 \mathrm{~d}$ & $5.25 \mathrm{c}$ & $5.00 \mathrm{c}$ & $5.00 \mathrm{c}$ & $5.00 \mathrm{c}$ & $4.00 \mathrm{~d}$ & $4.75 c$ \\
\hline \multirow[t]{2}{*}{ Mean } & $5.40 \mathrm{c}$ & $580 \mathrm{~b}$ & $5.20 \mathrm{c}$ & $6.40 \mathrm{a}$ & & $4.80 \mathrm{c}$ & $5.20 \mathrm{~b}$ & $5.00 \mathrm{~b}$ & $5.40 \mathrm{a}$ & \\
\hline & \multicolumn{5}{|c|}{ Root volume $\left(\mathrm{cm}^{3}\right)$} & \multicolumn{5}{|c|}{ Root volume $\left(\mathrm{cm}^{3}\right)$} \\
\hline Control & $3.6 \mathrm{cde}$ & $4.5 \mathrm{a}$ & $4 b c$ & $4 b c$ & $4.03 a$ & $2.27 \mathrm{~b}-\mathrm{e}$ & $3.27 \mathrm{a}$ & $2.9 \mathrm{ab}$ & $2.5 \mathrm{bcd}$ & $2.73 \mathrm{a}$ \\
\hline $1000 \mathrm{ppm}$ & $3 f g$ & $3 f g$ & $4 b c$ & $4.17 \mathrm{ab}$ & $3.54 \mathrm{~b}$ & $2.43 \mathrm{bcd}$ & $2.17 \mathrm{cde}$ & $2.90 \mathrm{ab}$ & $2.67 \mathrm{abc}$ & $2.54 \mathrm{ab}$ \\
\hline 2000ppm & $3.27 \mathrm{ef}$ & $4.5 \mathrm{a}$ & $3 f g$ & $2.5 \mathrm{~h}$ & $3.32 b$ & $2.27 b-e$ & $3.17 \mathrm{a}$ & $2.4 \mathrm{bcd}$ & $1.73 \mathrm{ef}$ & $2.39 \mathrm{~b}$ \\
\hline $3000 \mathrm{ppm}$ & $3 \mathrm{fg}$ & $3.27 \mathrm{ef}$ & $2.67 \mathrm{gh}$ & $3.50 \mathrm{de}$ & $3.11 \mathrm{c}$ & $2.17 \mathrm{c}-\mathrm{e}$ & $2.43 \mathrm{bcd}$ & $2 \mathrm{def}$ & $2.5 \mathrm{bcd}$ & $2.28 b$ \\
\hline $4000 \mathrm{ppm}$ & $3.70 \mathrm{~cd}$ & $4 b c$ & $3 \mathrm{fg}$ & $2 \mathrm{i}$ & $3.18 \mathrm{c}$ & 2def & $2.7 \mathrm{abc}$ & $1.67 \mathrm{ef}$ & $1.5 f$ & $1.97 \mathrm{c}$ \\
\hline \multirow[t]{2}{*}{ Mean } & $3.31 \mathrm{~b}$ & $3.85 \mathrm{a}$ & $3.33 b$ & $3.23 b$ & & $2.23 b c$ & $2.75 \mathrm{a}$ & $2.37 b$ & $2.18 \mathrm{c}$ & \\
\hline & \multicolumn{5}{|c|}{ Root fresh weight (g/ plant) } & \multicolumn{5}{|c|}{ Root fresh weight (g/ plant) } \\
\hline Control & $2.43 b-d$ & $2.17 \mathrm{cde}$ & $2.9 \mathrm{ab}$ & $2.67 \mathrm{a}-\mathrm{c}$ & $2.54 \mathrm{ab}$ & $2 \mathrm{jk}$ & $2.06 \mathrm{ijk}$ & $3.06 \mathrm{~b}-\mathrm{d}$ & $2.78 \mathrm{c}-\mathrm{e}$ & $2.48 b$ \\
\hline $1000 \mathrm{ppm}$ & $2.27 \mathrm{~b}-\mathrm{e}$ & $3.27 \mathrm{a}$ & $2.9 \mathrm{ab}$ & $2.5 b-d$ & $2.73 a$ & $2.54 \mathrm{e}-\mathrm{i}$ & $3.66 \mathrm{a}$ & $2.66 \mathrm{c}-\mathrm{g}$ & $3.29 \mathrm{ab}$ & $3.04 \mathrm{a}$ \\
\hline 2000ppm & $2.27 \mathrm{~b}-\mathrm{e}$ & $3.17 \mathrm{a}$ & $2.4 b-d$ & $1.73 \mathrm{ef}$ & $2.39 b$ & $1.93 \mathrm{jk}$ & $2.77 \mathrm{cde}$ & $1.73 \mathrm{k}$ & $2.19 \mathrm{f}-\mathrm{k}$ & $2.16 \mathrm{c}$ \\
\hline 3000ppm & $2.17 \mathrm{c}-\mathrm{e}$ & $2.43 b-d$ & $2 \mathrm{def}$ & $2.5 b-d$ & $2.28 \mathrm{~b}$ & $2.17 \mathrm{~g}-\mathrm{k}$ & $2.69 c-f$ & $2.39 \mathrm{e}-\mathrm{j}$ & $2.56 \mathrm{~d}-\mathrm{i}$ & $2.45 b$ \\
\hline $4000 \mathrm{ppm}$ & 2 def & $2.7 \mathrm{a}-\mathrm{c}$ & $1.67 \mathrm{ef}$ & $1.5 f$ & $1.97 \mathrm{c}$ & $2.39 \mathrm{e}-\mathrm{j}$ & $3.07 b c$ & $2.58 \mathrm{c}-\mathrm{h}$ & $2.13 \mathrm{~h}-\mathrm{k}$ & $2.54 \mathrm{~b}$ \\
\hline \multirow[t]{2}{*}{ Mean } & $2.22 b c$ & $2.75 \mathrm{a}$ & $2.37 b$ & $2.18 \mathrm{c}$ & & $2.21 \mathrm{c}$ & $2.85 \mathrm{a}$ & $2.49 \mathrm{~b}$ & $2.59 \mathrm{~b}$ & \\
\hline & \multicolumn{5}{|c|}{ Root dry weight (g/ plant) } & \multicolumn{5}{|c|}{ Root dry weight (g/ plant) } \\
\hline Control & $1.53 \mathrm{~g}-\mathrm{i}$ & $2.76 \mathrm{a}$ & $2.17 b-d$ & $1.92 \mathrm{~d}-\mathrm{f}$ & $2.10 \mathrm{a}$ & $1.28 \mathrm{fg}$ & $1.18 \mathrm{~g}-\mathrm{i}$ & $1.85 \mathrm{a}$ & $1.71 \mathrm{c}$ & $1.51 \mathrm{~b}$ \\
\hline $1000 \mathrm{ppm}$ & $1.97 \mathrm{c}-\mathrm{e}$ & $2.82 \mathrm{a}$ & $1.77 \mathrm{efg}$ & $2.32 \mathrm{~b}$ & $2.22 \mathrm{a}$ & $1.50 \mathrm{~d}$ & $1.86 \mathrm{a}$ & $1.28 \mathrm{fg}$ & $1.80 \mathrm{abc}$ & $1.61 \mathrm{a}$ \\
\hline 2000ppm & $1.55 \mathrm{~g}-\mathrm{i}$ & $2.85 \mathrm{a}$ & $1.37 \mathrm{i}$ & $1.93 \mathrm{~d}-\mathrm{f}$ & $1.93 \mathrm{~b}$ & $1.16 \mathrm{~g}-\mathrm{i}$ & $1.37 \mathrm{ef}$ & $1.01 \mathrm{jk}$ & $1.85 \mathrm{ab}$ & $1.35 \mathrm{c}$ \\
\hline $3000 \mathrm{ppm}$ & 1.44hi & $1.75 \mathrm{e}-\mathrm{g}$ & $1.73 \mathrm{e}-\mathrm{g}$ & $2.22 \mathrm{bc}$ & $1.79 \mathrm{c}$ & $1.07 \mathrm{ij}$ & $1.47 \mathrm{de}$ & $1.36 \mathrm{ef}$ & $1.28 \mathrm{fg}$ & $1.29 \mathrm{~cd}$ \\
\hline 4000ppm & $1.77 \mathrm{e}-\mathrm{g}$ & $1.34 \mathrm{i}$ & $1.67 \mathrm{f}-\mathrm{h}$ & $1.87 \mathrm{ef}$ & $1.66 \mathrm{c}$ & 1.13hi & $1.73 b c$ & $1.22 \mathrm{gh}$ & $0.94 \mathrm{k}$ & $1.25 \mathrm{~d}$ \\
\hline Mean & $1.65 \mathrm{~d}$ & $2.31 \mathrm{a}$ & $1.74 \mathrm{c}$ & $2.05 b$ & & $1.23 c$ & $1.52 \mathrm{a}$ & $1.34 \mathrm{~b}$ & $1.52 \mathrm{a}$ & \\
\hline
\end{tabular}

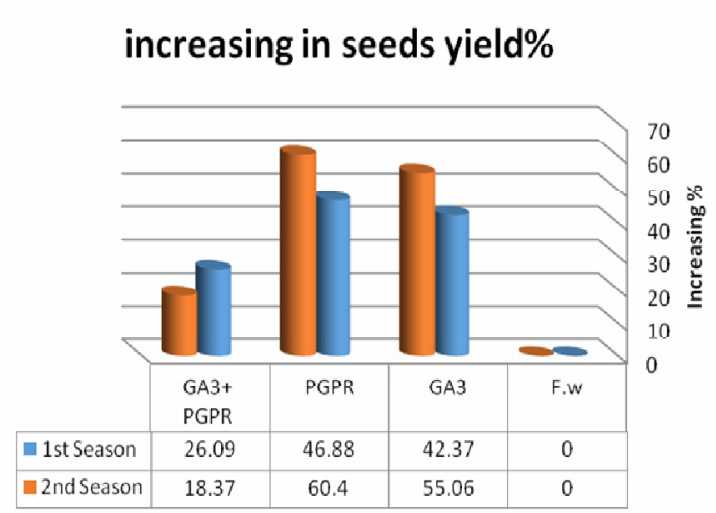

\section{Reduction in seeds yield\%}

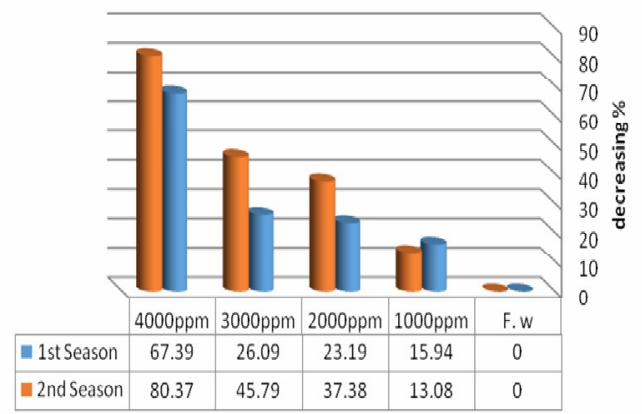

Figure.1. Reduction and increasing \% in seeds yield /plant as affected by salinity irrigation water levels and foliar application in the two seasons 
Table 3. Effect of saline irrigation water levels and spraying by $\mathrm{GA}_{3}$ and PGPR on capsules dry weight, seeds yield/plant (g) and weight of 1000 seed of Nigella sativa L. plants

\begin{tabular}{|c|c|c|c|c|c|c|c|c|c|c|}
\hline \multirow{3}{*}{$\begin{array}{l}\text { Seasons } \\
\text { Spraying } \\
\text { Salinity }\end{array}$} & \multicolumn{5}{|c|}{$1^{\text {st }}$ Season 2015} & \multicolumn{5}{|c|}{$2^{\text {nd }}$ Season 2016} \\
\hline & Fresh wate & $\mathbf{G A}_{3}$ & PGPR & $\begin{array}{l}\mathbf{G A}_{3}+ \\
\text { PGPR }\end{array}$ & Mean & Fresh water & $\mathbf{G A}_{3}$ & PGPR & $\begin{array}{l}\mathbf{G A}_{3}+ \\
\text { PGPR }\end{array}$ & Mean \\
\hline & \multicolumn{5}{|c|}{ Capsules dry weight /plant (g) } & \multicolumn{5}{|c|}{ Capsules dry weight /plant (g) } \\
\hline Control & $2.45 \mathrm{~g}$ & $3.03 \mathrm{de}$ & $3.54 a-c$ & $4.02 \mathrm{a}$ & $3.26 \mathrm{a}$ & $1.95 \mathrm{de}$ & $2.46 b c$ & $2.44 \mathrm{bc}$ & $3.01 \mathrm{a}$ & $2.47 \mathrm{a}$ \\
\hline 1000ppm & $1.65 \mathrm{~h}$ & $2.77 \mathrm{~d}-\mathrm{g}$ & $3.74 \mathrm{ab}$ & $3.26 \mathrm{~b}-\mathrm{d}$ & $2.85 b c$ & $1.09 \mathrm{~h}$ & $1.64 \mathrm{e}-\mathrm{g}$ & $3.03 \mathrm{a}$ & $2.63 \mathrm{ab}$ & $2.10 \mathrm{bc}$ \\
\hline 2000ppm & $1.83 \mathrm{~h}$ & $2.67 \mathrm{e}-\mathrm{g}$ & $2.92 \mathrm{~d}-\mathrm{g}$ & $3.75 \mathrm{ab}$ & $2.79 \mathrm{c}$ & $1.49 \mathrm{f}-\mathrm{h}$ & $1.56 \mathrm{e}-\mathrm{g}$ & $2.19 \mathrm{~cd}$ & $2.77 \mathrm{ab}$ & $2.00 \mathrm{~cd}$ \\
\hline 3000ppm & $2.50 \mathrm{~g}$ & $3.02 \mathrm{~d}-\mathrm{f}$ & $3.58 \mathrm{a}-\mathrm{c}$ & $3.09 \mathrm{c}-\mathrm{e}$ & $3.05 \mathrm{ab}$ & $1.89 \mathrm{~d}-\mathrm{f}$ & $1.71 \mathrm{e}-\mathrm{g}$ & $2.82 \mathrm{ab}$ & $2.44 \mathrm{bc}$ & $2.21 \mathrm{~b}$ \\
\hline 4000ppm & $2.51 \mathrm{fg}$ & $2.48 \mathrm{~g}$ & $3.72 \mathrm{ab}$ & $2.42 \mathrm{~g}$ & $2.78 \mathrm{c}$ & $1.60 \mathrm{e}-\mathrm{g}$ & $1.61 \mathrm{e}-\mathrm{g}$ & $2.72 \mathrm{ab}$ & $1.45 \mathrm{gh}$ & $1.85 \mathrm{~d}$ \\
\hline \multirow[t]{2}{*}{ Mean } & $2.19 \mathrm{~d}$ & $2.79 \mathrm{c}$ & $3.50 \mathrm{a}$ & $3.31 \mathrm{~b}$ & & $1.60 \mathrm{~d}$ & $1.80 \mathrm{c}$ & $2.64 \mathrm{a}$ & $2.46 \mathrm{~b}$ & \\
\hline & \multicolumn{5}{|c|}{ Seeds yield/plant $(\mathrm{g})$} & \multicolumn{5}{|c|}{ Seeds yield/plant (g ) } \\
\hline Control & $0.79 \mathrm{~h}$ & $1.83 \mathrm{a}$ & $1.56 \mathrm{c}$ & $1.32 \mathrm{e}$ & $1.38 \mathrm{a}$ & $0.58 \mathrm{ef}$ & $1.46 \mathrm{a}$ & $1.35 \mathrm{~b}$ & $0.88 \mathrm{~d}$ & $1.07 \mathrm{a}$ \\
\hline $1000 \mathrm{ppm}$ & $0.93 \mathrm{~g}$ & $1.36 \mathrm{de}$ & $1.64 b$ & $0.70 \mathrm{i}$ & $1.16 \mathrm{~b}$ & $0.61 \mathrm{e}$ & $1.21 \mathrm{c}$ & $1.41 \mathrm{ab}$ & $0.50 \mathrm{fg}$ & $0.93 b$ \\
\hline 2000ppm & $0.85 \mathrm{~h}$ & $1.59 \mathrm{bc}$ & $1.20 \mathrm{f}$ & $0.61 \mathrm{jk}$ & $1.06 \mathrm{c}$ & $0.42 \mathrm{gh}$ & $1.15 \mathrm{c}$ & $0.87 \mathrm{~d}$ & $0.23 \mathrm{jk}$ & $0.67 \mathrm{c}$ \\
\hline 3000ppm & 0.57 & 0.461 & $1.63 \mathrm{bc}$ & $1.40 \mathrm{~d}$ & $1.02 \mathrm{~d}$ & $0.28 \mathrm{ij}$ & $0.29 \mathrm{ij}$ & $1.17 \mathrm{c}$ & $0.58 \mathrm{ef}$ & $0.58 \mathrm{~d}$ \\
\hline 4000ppm & $0.24 n$ & $0.66 \mathrm{ij}$ & $0.35 \mathrm{~m}$ & $0.57 \mathrm{k}$ & $0.45 \mathrm{e}$ & 0.121 & $0.34-\mathrm{i}$ & $0.23 \mathrm{jk}$ & $0.24 \mathrm{jk}$ & $0.21 \mathrm{e}$ \\
\hline \multirow[t]{2}{*}{ Mean } & $0.68 \mathrm{~d}$ & $1.18 \mathrm{~b}$ & $1.28 \mathrm{a}$ & $0.92 \mathrm{c}$ & & $0.40 \mathrm{~d}$ & $0.89 b$ & $1.01 \mathrm{a}$ & $0.49 \mathrm{c}$ & \\
\hline & \multicolumn{5}{|c|}{ Weight of 1000 seed $(\mathrm{g})$} & \multicolumn{5}{|c|}{ Weight of 1000 seed (g ) } \\
\hline Control & $3.07 \mathrm{~b}$ & $3.59 \mathrm{a}$ & $2.61 \mathrm{c}$ & $2.34 \mathrm{~d}-\mathrm{g}$ & $2.90 \mathrm{a}$ & $2.65 b$ & $2.83 \mathrm{a}$ & $2.22 \mathrm{c}$ & $2.23 \mathrm{c}$ & $2.48 \mathrm{a}$ \\
\hline 1000ppm & 2.45 & $2.47 \mathrm{c}-\mathrm{e}$ & $2.49 \mathrm{~cd}$ & $2.25 \mathrm{f}-\mathrm{h}$ & $2.42 b$ & $2.17 \mathrm{~cd}$ & $2.22 \mathrm{c}$ & $2.17 \mathrm{~cd}$ & $2.13 \mathrm{~cd}$ & $2.17 b$ \\
\hline 2000ppm & $2.37 \mathrm{~d}-\mathrm{g}$ & $2.44 \mathrm{de}$ & $2.33 \mathrm{e}-\mathrm{g}$ & $2.14 \mathrm{~h}$ & $2.32 \mathrm{c}$ & $2.11 \mathrm{~cd}$ & $2.14 \mathrm{~cd}$ & $2.13 \mathrm{~cd}$ & $2.08 \mathrm{~d}$ & $2.11 \mathrm{c}$ \\
\hline 3000ppm & $2.23 \mathrm{gh}$ & $2.39 \mathrm{~d}-\mathrm{f}$ & $2.18 \mathrm{~h}$ & $2.15 \mathrm{~h}$ & $2.24 \mathrm{~d}$ & $2.06 \mathrm{~d}$ & $2.13 \mathrm{~cd}$ & $2.08 \mathrm{~d}$ & $1.91 \mathrm{e}$ & $2.05 \mathrm{~d}$ \\
\hline 4000ppm & $1.12 \mathrm{k}$ & $1.53 \mathrm{j}$ & $1.73 \mathrm{i}$ & $1.83 \mathrm{i}$ & $1.56 \mathrm{e}$ & $1.12 \mathrm{~h}$ & $1.42 \mathrm{~g}$ & $1.46 \mathrm{~g}$ & $1.59 \mathrm{f}$ & $1.40 \mathrm{e}$ \\
\hline Mean & $2.25 \mathrm{~b}$ & $2.49 \mathrm{a}$ & $2.27 \mathrm{~b}$ & $2.15 \mathrm{c}$ & & $2.02 \mathrm{~b}$ & $2.15 \mathrm{a}$ & $2.01 \mathrm{~b}$ & $1.99 \mathrm{~b}$ & \\
\hline
\end{tabular}

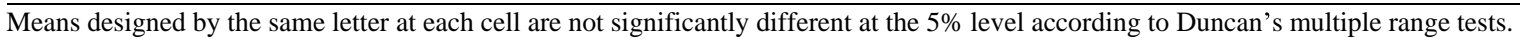
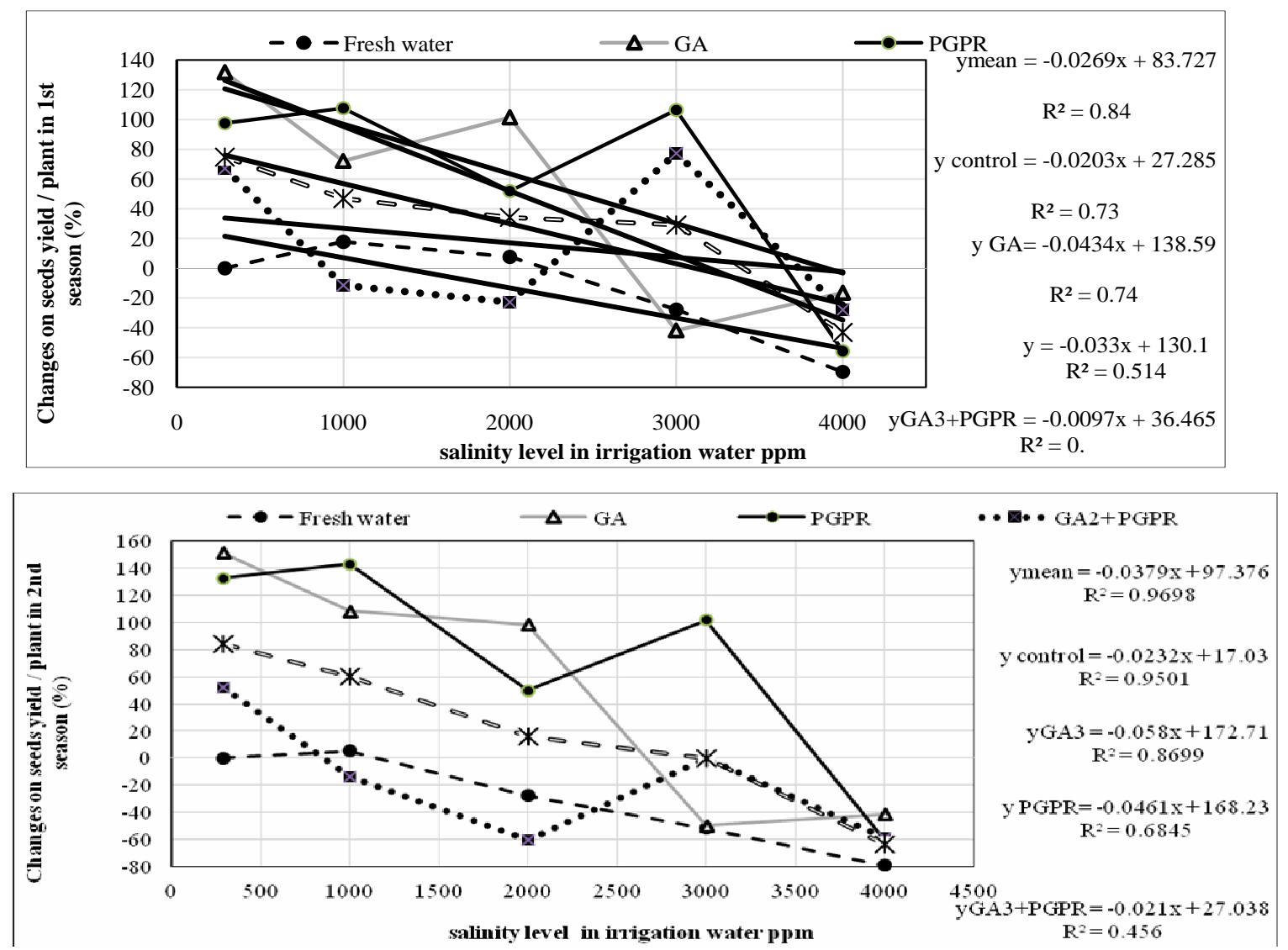

Figure 2. Correlation of seeds yield /plant of black cumin as affected by salinity irrigation water levels and foliar application of $\mathrm{GA}_{3}$ and PGPR 
Table 4. Effect of saline irrigation water levels and spraying by GA $\mathrm{A}_{3}$ and PGPR on chlorophyll (a) mg/g F. W., chlorophyll (b) $\mathrm{mg} / \mathrm{g} \mathrm{F}$. W, relative water content and membrane permeability \% of Nigella sativa L. plants

\begin{tabular}{|c|c|c|c|c|c|c|c|c|c|c|}
\hline \multirow{2}{*}{$\begin{array}{l}\text { Seasons } \\
\text { Spraying } \\
\text { Salinity } \\
\end{array}$} & \multicolumn{5}{|c|}{$1^{\text {st }}$ Season 2015} & \multicolumn{5}{|c|}{$2^{\text {nd }}$ Season 2016} \\
\hline & $\begin{array}{l}\text { Fresh } \\
\text { water }\end{array}$ & $\mathbf{G A}_{3}$ & PGPR & $\begin{array}{l}\text { GA }_{3}+ \\
\text { PGPR }\end{array}$ & Mean & Fresh wate & el $\mathbf{G A}_{3}$ & PGPR & $\begin{array}{l}\text { GA }_{3}+ \\
\text { PGPR }\end{array}$ & Mean \\
\hline & \multicolumn{5}{|c|}{ Chlorophyll( a) mg/g F. W } & \multicolumn{5}{|c|}{ Chlorophyll( a) mg/g F. W } \\
\hline Control & $1.50 f$ & $1.70 \mathrm{a}$ & $1.51 \mathrm{fg}$ & $1.60 \mathrm{~cd}$ & $1.58 \mathrm{a}$ & $1.46 \mathrm{ef}$ & $1.32 \mathrm{i}$ & $1.45 \mathrm{ef}$ & $1.53 \mathrm{a}$ & $1.44 \mathrm{a}$ \\
\hline 1000ppm & $1.40 \mathrm{k}$ & $1.60 \mathrm{~cd}$ & $1.52 \mathrm{f}$ & $1.55 \mathrm{e}$ & $1.52 b$ & $1.47 \mathrm{de}$ & $1.35 \mathrm{i}$ & $1.48 \mathrm{~cd}$ & $1.51 \mathrm{ab}$ & $1.45 \mathrm{a}$ \\
\hline 2000ppm & $1.57 \mathrm{~d}$ & $1.41 \mathrm{jk}$ & $1.55 \mathrm{e}$ & $1.61 b c$ & $1.54 \mathrm{~b}$ & $1.42 \mathrm{gh}$ & $1.44 f g$ & $1.44 \mathrm{fg}$ & $1.40 \mathrm{~h}$ & $1.42 b$ \\
\hline $3000 \mathrm{ppm}$ & $1.44 \mathrm{ij}$ & $1.52 \mathrm{f}$ & $1.63 b$ & $1.55 \mathrm{e}$ & $1.53 \mathrm{c}$ & $1.33 \mathrm{i}$ & $1.51 \mathrm{ab}$ & $1.34 \mathrm{i}$ & $1.50 \mathrm{bc}$ & $1.42 \mathrm{~b}$ \\
\hline 4000ppm & $1.49 \mathrm{~g}$ & $1.51 \mathrm{fg}$ & $1.50 \mathrm{fg}$ & 1.46hi & $1.49 \mathrm{~d}$ & $1.33 \mathrm{i}$ & $1.49 \mathrm{bc}$ & $1.45 \mathrm{ef}$ & $1.35 \mathrm{i}$ & $1.41 \mathrm{c}$ \\
\hline \multirow[t]{2}{*}{ Mean } & $1.48 \mathrm{c}$ & $1.55 \mathrm{ab}$ & $1.54 \mathrm{~b}$ & $1.55 \mathrm{a}$ & & $1.40 \mathrm{c}$ & $1.42 \mathrm{~b}$ & $1.43 \mathrm{~b}$ & $1.46 \mathrm{a}$ & \\
\hline & \multicolumn{5}{|c|}{ Chlorophyll( b) mg/g F. W } & \multicolumn{5}{|c|}{ Chlorophyll( b) mg/g F. W } \\
\hline Control & $0.71 \mathrm{e}$ & $0.90 \mathrm{a}$ & $0.75 \mathrm{~cd}$ & $0.76 \mathrm{c}$ & $0.78 \mathrm{a}$ & $0.68 \mathrm{de}$ & $0.77 \mathrm{a}$ & $0.63 \mathrm{~g}$ & $0.74 b$ & $0.71 \mathrm{a}$ \\
\hline 1000ppm & $0.73 \mathrm{de}$ & $0.66 \mathrm{f}$ & $0.65 \mathrm{f}$ & $0.80 \mathrm{~b}$ & $0.71 b$ & $0.76 \mathrm{a}$ & $0.67 \mathrm{ef}$ & $0.40 \mathrm{~m}$ & $0.71 \mathrm{c}$ & $0.64 b$ \\
\hline 2000ppm & $0.82 b$ & $0.76 \mathrm{c}$ & $0.47 \mathrm{i}$ & $0.81 b$ & $0.71 b$ & $0.69 \mathrm{~d}$ & 0.461 & $0.56 \mathrm{i}$ & $0.74 b$ & $0.61 \mathrm{c}$ \\
\hline $3000 \mathrm{ppm}$ & $0.44 \mathrm{j}$ & $0.74 \mathrm{~cd}$ & $0.61 \mathrm{~g}$ & $0.80 \mathrm{~b}$ & $0.65 \mathrm{c}$ & $0.67 f$ & $0.40 \mathrm{~m}$ & $0.50 \mathrm{k}$ & $0.72 \mathrm{c}$ & $0.57 \mathrm{~d}$ \\
\hline 4000ppm & $0.71 \mathrm{e}$ & $0.43 \mathrm{j}$ & $0.58 \mathrm{~h}$ & $0.81 b$ & $0.63 d$ & $0.39 \mathrm{~m}$ & $0.61 \mathrm{~h}$ & $0.53 \mathrm{j}$ & $0.71 \mathrm{c}$ & $0.56 \mathrm{e}$ \\
\hline \multirow[t]{2}{*}{ Mean } & $0.68 \mathrm{c}$ & $0.70 \mathrm{~b}$ & $0.61 \mathrm{~d}$ & $0.80 \mathrm{a}$ & & $0.64 b$ & $0.58 \mathrm{c}$ & $0.52 \mathrm{~d}$ & $0.72 \mathrm{a}$ & \\
\hline & \multicolumn{5}{|c|}{ Relative water content (RWC)\% } & \multicolumn{5}{|c|}{ Relative water content (RWC)\% } \\
\hline Control & $77.66 \mathrm{e}$ & $86.56 b$ & $81.38 \mathrm{c}$ & $90.46 \mathrm{a}$ & $84.02 \mathrm{a}$ & $76.52 \mathrm{e}$ & $85.44 \mathrm{~b}$ & $80.59 \mathrm{c}$ & $89.41 \mathrm{a}$ & $82.99 \mathrm{a}$ \\
\hline $1000 \mathrm{ppm}$ & 66.451 & $76.34 f$ & $78.42 d$ & $75.33 \mathrm{~g}$ & $74.14 b$ & 65.301 & $75.40 \mathrm{e}$ & $77.44 d$ & $74.41 \mathrm{~g}$ & $73.14 b$ \\
\hline 2000ppm & $65.46 \mathrm{~m}$ & $74.10 \mathrm{~h}$ & $77.70 \mathrm{e}$ & $73.33 \mathrm{i}$ & $72.64 \mathrm{c}$ & $64.41 \mathrm{~m}$ & $73.15 h$ & $76.41 \mathrm{e}$ & $72.48 \mathrm{i}$ & $71.61 \mathrm{c}$ \\
\hline 3000 ppm & 63.610 & $72.60 \mathrm{j}$ & $78.42 \mathrm{~d}$ & $64.54 n$ & $69.74 d$ & $62.52 \mathrm{o}$ & $71.26 \mathrm{j}$ & $77.41 d$ & $63.40 \mathrm{n}$ & $68.65 d$ \\
\hline 4000ppm & $56.48 \mathrm{q}$ & $68.58 \mathrm{k}$ & $77.46 \mathrm{e}$ & $62.57 \mathrm{p}$ & $66.27 \mathrm{e}$ & $55.55 q$ & $67.45 \mathrm{k}$ & $76.37 \mathrm{e}$ & $61.33 p$ & $65.18 \mathrm{e}$ \\
\hline \multirow[t]{2}{*}{ Mean } & $65.89 \mathrm{~d}$ & $75.64 b$ & $78.68 \mathrm{a}$ & $73.25 \mathrm{c}$ & & $64.86 \mathrm{~d}$ & $74.54 b$ & $77.65 \mathrm{a}$ & $72.21 \mathrm{c}$ & \\
\hline & \multicolumn{5}{|c|}{ Membrane permeability (MP) \% } & \multicolumn{5}{|c|}{ Membrane permeability(MP)\% } \\
\hline Control & $68.28 \mathrm{j}$ & $73.81 \mathrm{~h}$ & 43.311 & $32.05 \mathrm{~m}$ & $54.37 \mathrm{e}$ & $56.96 \mathrm{j}$ & $70.34 f$ & 40.471 & $32.11 \mathrm{~m}$ & $49.97 d$ \\
\hline $1000 \mathrm{ppm}$ & $72.79 \mathrm{~h}$ & $91.21 \mathrm{~b}$ & $63.61 \mathrm{k}$ & $77.01 \mathrm{~g}$ & $76.15 \mathrm{~d}$ & $60.93 \mathrm{i}$ & $80.04 d$ & $60.19 \mathrm{i}$ & $69.85 f$ & $67.76 \mathrm{c}$ \\
\hline 2000ppm & $89.03 c$ & $91.63 \mathrm{~b}$ & $73.99 \mathrm{~h}$ & $88.57 \mathrm{c}$ & $85.81 \mathrm{~b}$ & $63.70 \mathrm{~h}$ & $89.89 a$ & $67.92 \mathrm{~g}$ & $86.28 b$ & $76.95 b$ \\
\hline 3000ppm & $70.84 \mathrm{i}$ & $86.02 \mathrm{~d}$ & $86.00 \mathrm{~d}$ & $94.64 \mathrm{a}$ & $84.38 \mathrm{~d}$ & $54.07 \mathrm{k}$ & $80.28 d$ & $82.68 \mathrm{c}$ & $90.41 \mathrm{a}$ & $76.86 \mathrm{~b}$ \\
\hline 4000ppm & $81.97 \mathrm{e}$ & $95.16 \mathrm{a}$ & $79.09 f$ & $92.16 b$ & $87.10 \mathrm{a}$ & $57.20 \mathrm{j}$ & $91.85 \mathrm{a}$ & $72.61 \mathrm{e}$ & $90.50 \mathrm{a}$ & $78.04 \mathrm{a}$ \\
\hline Mean & $76.58 b$ & $87.57 \mathrm{a}$ & $69.20 \mathrm{c}$ & $76.89 \mathrm{~b}$ & & $58.57 \mathrm{~d}$ & $82.48 \mathrm{a}$ & $64.77 \mathrm{c}$ & $73.83 b$ & \\
\hline
\end{tabular}

Means designed by the same letter at each cell are not significantly different at the 5\% level according to Duncan's multiple range tests.

when $\mathrm{GA}_{3}$ was used without salinity treatment in both growth seasons.

The relationship between saline irrigation water levels and spraying $\mathrm{GA}_{3}$ and PGPR on changes in seeds yield in both seasons.

A positive linear relationship was obtained between saline irrigation water levels and spraying $\mathrm{GA}_{3}$ and PGPR on seeds yield changes in both seasons (Figure 2 ). The correlation coefficient values $\mathrm{r}^{2} 0.74,0.74,0.51$, and 0.08 , respectively in the first season and $0.95,0.87$, 0.68 , and 0.46 , respectively in the second season. The positive relationship indicated that there is a high reduction in seeds yield with increasing salinity level, this reduction reduced by spraying treatments especially $\mathrm{GA}_{3}$ in both seasons. At low saline irrigation level, there is an increase in seeds yield reached 131.65 and $151.72 \%$ with using $\mathrm{GA}_{3}$ in both seasons, successively.

\section{Biochemical characteristics}

Increasing salinity levels from 0 to $4000 \mathrm{ppm}$ caused a gradual decrease in chlorophyll a, chlorophyll b, fixed oil and RWC in Nigella sativa L. Table (4 and 5). The most elevated salinity level recorded the least values in this respect. Furthermore, membrane permeability (Mp), proline accumulation, (CAT) and (POX) enzymes activities were gradually increased by increasing salinity concentrations. Spraying $\mathrm{GA}_{3}+$ PGPR noticeably increased chlorophyll a and b content, fixed oil\%, proline accumulation, CAT and POX enzymes activity in both seasons, while using $\mathrm{GA}_{3}$ increased membrane permeability in both seasons. The promotion effect was observed when salinity levels were combined with $\mathrm{GA}_{3}$ or $\mathrm{GA}_{3}+\mathrm{PGPR}$ treatments. The greatest 
chlorophyll a and $b$ were recorded by $\mathrm{GA}_{3}$ without salinity for both seasons.

Furthermore, spraying $\mathrm{GA}_{3}+$ PGPR without salinity recorded the maximum fixed oil percentage. Proline accumulation, CAT and POX enzymes activities

were pronounced when salinity treatments were combined with $\mathrm{GA}_{3}+$ PGPR treatments and the greatest values were noted by 4000 ppm salinity level with $\mathrm{GA}_{3}$ + PGPR. However, when salinity treatments combined with $\mathrm{GA}_{3}$ or PGPR treatments, the lessening in RWC was retarded and the highest membrane permeability recorded at 3000 and 4000 ppm together with $\mathrm{GA}_{3}$ or $\mathrm{GA}_{3}+\mathrm{PGPR}$ treatments in both seasons.

\section{Total soluble salts}

Fig (3) show a positive linear relationship obtained between used saline water and spraying $\mathrm{GA}_{3}, \mathrm{PGPR}$ and $\mathrm{GA}_{3}+\mathrm{PGPR}$ on values of soil EC. There are highly significant (with correlation coefficient values, $\mathrm{r}^{2}=0.95$, 0.97, 0.92, and 0.97 for spraying with $\mathrm{GA}_{3}$, PGPR, and $\mathrm{GA}_{3}+\mathrm{PGPR}$, respectively). Moreover, significant variations in the values of soil EC after using different saline irrigation water, which increased significantly in excess of salinity concentrations of 2000 ppm, 3000 ppm and 4000 ppm diluted sea water for irrigation.

Table 5. Effect of saline irrigation water levels and treatment with $\mathrm{GA}_{3}$ or PGPR on fixed oil\%, proline and antioxidant enzyme activities of Nigella sativa $\mathrm{L}$. plants

\begin{tabular}{|c|c|c|c|c|c|c|c|c|c|c|}
\hline \multirow{2}{*}{$\begin{array}{l}\text { Seasons } \\
\text { Spraying } \\
\text { Salinity }\end{array}$} & \multicolumn{5}{|c|}{$1^{\text {st }}$ Season 2015} & \multicolumn{5}{|c|}{$2^{\text {nd }}$ Season 2016} \\
\hline & resh wat & $\mathbf{G A}_{3}$ & PGPR & $\begin{array}{l}\mathrm{GA}_{3}+ \\
\text { PGPR }\end{array}$ & Mean & $\begin{array}{l}\text { Fresh } \\
\text { water }\end{array}$ & $\mathbf{G A}_{3}$ & PGPR & $\begin{array}{l}\mathrm{GA}_{3}+ \\
\text { PGPR }\end{array}$ & Mean \\
\hline & \multicolumn{5}{|c|}{ Fixed oil\% } & \multicolumn{5}{|c|}{ Fixed oil\% } \\
\hline Control & $30.86 \mathrm{~h}$ & $34.11 \mathrm{e}$ & $39.53 b$ & $40.13 a$ & $36.16 \mathrm{a}$ & $29.00 f g$ & $32.74 d$ & $37.04 \mathrm{~b}$ & $38.33 \mathrm{a}$ & \\
\hline 1000ppm & $31.20 \mathrm{~g}$ & $31.94 \mathrm{~g}$ & $39.05 \mathrm{c}$ & $39.72 b$ & $35.48 b$ & $30.34 \mathrm{e}$ & $29.69 \mathrm{ef}$ & $35.56 \mathrm{c}$ & $36.22 b c$ & \\
\hline 2000ppm & $29.58 \mathrm{i}$ & 26.381 & $28.51 \mathrm{j}$ & $36.28 d$ & $30.19 \mathrm{c}$ & $28.52 \mathrm{~g}$ & $24.00 \mathrm{j}$ & $27.23 \mathrm{~h}$ & $30.37 \mathrm{e}$ & \\
\hline 3000ppm & $17.56 \mathrm{p}$ & $23.48 n$ & $27.51 \mathrm{k}$ & $32.88 \mathrm{f}$ & $25.36 \mathrm{~d}$ & $16.50 \mathrm{n}$ & 21.561 & $25.89 \mathrm{i}$ & $30.34 \mathrm{e}$ & \\
\hline 4000ppm & $13.49 \mathrm{q}$ & 22.920 & $24.52 \mathrm{~m}$ & $29.35 \mathrm{i}$ & $22.57 \mathrm{e}$ & 12.730 & $19.56 \mathrm{~m}$ & $22.71 \mathrm{k}$ & $27.48 \mathrm{~h}$ & \\
\hline \multirow[t]{2}{*}{ Mean } & $24.54 \mathrm{~d}$ & $27.76 c$ & $31.82 \mathrm{~b}$ & $35.67 \mathrm{a}$ & & $23.42 d$ & $25.51 \mathrm{c}$ & $29.68 b$ & $32.55 \mathrm{a}$ & \\
\hline & \multicolumn{5}{|c|}{ Proline $\left(? \mathrm{~mol} / \mathrm{g}^{-1} \mathrm{FW}\right)$} & \multicolumn{5}{|c|}{ Proline $\left(? \mathrm{~mol} / \mathrm{g}^{-1} \mathrm{FW}\right)$} \\
\hline Control & $1.71 \mathrm{~h}$ & $2.34 \mathrm{f}$ & $2.56 \mathrm{e}^{\circ}$ & $2.67 \mathrm{de}$ & $2.32 \mathrm{~d}$ & $1.49 \mathrm{jk}$ & $1.88 \mathrm{gh}$ & $2.2 \mathrm{def}$ & $2.49 \mathrm{a}-\mathrm{c}$ & $2.02 \mathrm{~b}$ \\
\hline $1000 \mathrm{ppm}$ & $1.72 \mathrm{~h}$ & $2.09 \mathrm{~g}$ & $2.77 \mathrm{~d}$ & $3.12 \mathrm{c}$ & $2.42 \mathrm{c}$ & $1.61 \mathrm{ij}$ & $1.89 \mathrm{gh}$ & $2.1 \mathrm{fg}$ & $2.33 \mathrm{c}-\mathrm{e}$ & $1.98 \mathrm{bc}$ \\
\hline 2000ppm & $1.82 \mathrm{~h}$ & $2.14 \mathrm{~g}$ & $2.7 \mathrm{de}$ & $3.2 \mathrm{bc}$ & $2.47 \mathrm{bc}$ & $1.3 \mathrm{k}$ & $1.60 \mathrm{ij}$ & $2.1 \mathrm{fg}$ & $2.59 \mathrm{ab}$ & $1.90 \mathrm{c}$ \\
\hline 3000ppm & $1.66 \mathrm{~h}$ & $2.09 \mathrm{~g}$ & $3.29 \mathrm{ab}$ & $3.11 \mathrm{c}$ & $2.53 b$ & $1.38 \mathrm{k}$ & $1.89 \mathrm{gh}$ & $2.08 f g$ & $2.39 b-d$ & $1.93 \mathrm{bc}$ \\
\hline 4000ppm & $1.76 \mathrm{~h}$ & $2.16 \mathrm{~g}$ & $3.36 \mathrm{ab}$ & $3.39 \mathrm{a}$ & $2.67 \mathrm{a}$ & 1.76hi & $1.88 \mathrm{gh}$ & $2.15 \mathrm{ef}$ & $2.70 \mathrm{a}$ & $2.12 \mathrm{a}$ \\
\hline \multirow[t]{2}{*}{ Mean } & $1.73 \mathrm{~d}$ & $2.16 \mathrm{c}$ & $2.93 b$ & $3.09 \mathrm{a}$ & & $1.51 \mathrm{~d}$ & $1.83 \mathrm{c}$ & $2.13 b$ & $2.50 \mathrm{a}$ & \\
\hline & \multicolumn{9}{|c|}{ Antioxidant enzyme activities } & \\
\hline Control & 0.871 & $0.93 \mathrm{k}$ & $1.16 \mathrm{~h}$ & $1.44 \mathrm{f}$ & $1.10 \mathrm{e}$ & $0.77 \mathrm{j}$ & $0.87 \mathrm{hi}$ & $0.97 \mathrm{fg}$ & $1.18 \mathrm{~d}$ & $0.95 \mathrm{~d}$ \\
\hline 1000ppm & $0.96 \mathrm{jk}$ & $1.21 \mathrm{~h}$ & $1.58 \mathrm{de}$ & $1.86 \mathrm{~b}$ & $1.40 \mathrm{~cd}$ & $0.80 \mathrm{ij}$ & $0.92 \mathrm{gh}$ & $1.32 \mathrm{c}$ & $1.47 \mathrm{~b}$ & $1.13 \mathrm{c}$ \\
\hline 2000ppm & $0.98 \mathrm{ij}$ & $1.26 \mathrm{~g}$ & $1.59 \mathrm{de}$ & $1.90 \mathrm{ab}$ & $1.43 c$ & $0.87 \mathrm{hi}$ & $1.01 \mathrm{ef}$ & $1.32 \mathrm{c}$ & $1.59 \mathrm{a}$ & $1.19 b$ \\
\hline 3000ppm & $1.03 \mathrm{i}$ & $1.46 \mathrm{f}$ & $1.64 d$ & $1.88 \mathrm{~b}$ & $1.50 \mathrm{~b}$ & $0.92 \mathrm{gh}$ & $1.07 \mathrm{e}$ & $1.29 \mathrm{c}$ & $1.48 \mathrm{~b}$ & $1.19 b$ \\
\hline 4000ppm & $1.31 \mathrm{~g}$ & $1.57 \mathrm{e}$ & $1.74 \mathrm{c}$ & $1.95 \mathrm{a}$ & $1.64 \mathrm{a}$ & $1.04 \mathrm{ef}$ & $1.24 \mathrm{~cd}$ & $1.46 \mathrm{~b}$ & $1.62 \mathrm{a}$ & $1.34 \mathrm{a}$ \\
\hline \multirow[t]{2}{*}{ Mean } & $1.03 \mathrm{~d}$ & $1.28 \mathrm{c}$ & $1.54 \mathrm{~b}$ & $1.81 \mathrm{a}$ & & $0.88 \mathrm{~d}$ & $1.02 \mathrm{c}$ & $1.27 \mathrm{~b}$ & $1.47 \mathrm{a}$ & \\
\hline & \multicolumn{5}{|c|}{ POX ?mol min ${ }^{-1} \mathrm{mg}^{-1}$ protein } & \multicolumn{5}{|c|}{ POX ?mol min ${ }^{-1} \mathrm{mg}^{-1}$ protein } \\
\hline Control & 11.671 & $14.05 \mathrm{jk}$ & $15.19 \mathrm{~h}-\mathrm{j}$ & $17.88 f g$ & $14.69 \mathrm{e}$ & 11.071 & $13.52 \mathrm{i}-\mathrm{k}$ & $14.23 \mathrm{~h}-\mathrm{j}$ & $16.23 \mathrm{f}-\mathrm{h}$ & $13.76 \mathrm{~d}$ \\
\hline 1000ppm & $13.25 \mathrm{k}$ & $15.47 \mathrm{hi}$ & $18.48 \mathrm{e}-\mathrm{g}$ & $19.56 \mathrm{de}$ & $16.69 \mathrm{~d}$ & $12.14 \mathrm{kl}$ & $13.71 \mathrm{i}-\mathrm{k}$ & $17.34 \mathrm{~d}-\mathrm{f}$ & $18.29 \mathrm{de}$ & $15.37 \mathrm{c}$ \\
\hline 2000ppm & $14.30 \mathrm{i}-\mathrm{k}$ & $17.61 \mathrm{~g}$ & $20.52 d$ & $21.97 \mathrm{c}$ & $18.60 \mathrm{c}$ & $13.19 \mathrm{jk}$ & $16.15 f-h$ & $18.56 \mathrm{~d}$ & $20.84 c$ & $17.19 \mathrm{~b}$ \\
\hline 3000ppm & $15.61 \mathrm{~h}$ & $18.00 \mathrm{fg}$ & $22.48 \mathrm{c}$ & $25.45 b$ & $20.39 b$ & $14.34 \mathrm{~h}-\mathrm{j}$ & $16.52 \mathrm{e}-\mathrm{g}$ & $23.16 \mathrm{ab}$ & $22.59 b c$ & $19.16 \mathrm{a}$ \\
\hline 4000ppm & $17.41 \mathrm{~g}$ & $19.05 \mathrm{ef}$ & $24.86 b$ & $27.89 \mathrm{a}$ & $22.30 \mathrm{a}$ & $15.30 \mathrm{~g}-\mathrm{i}$ & $17.26 \mathrm{~d}-\mathrm{g}$ & $22.59 b c$ & $24.66 \mathrm{a}$ & $19.95 \mathrm{a}$ \\
\hline Mean & $14.44 \mathrm{~d}$ & $16.84 \mathrm{c}$ & $20.31 b$ & $22.55 \mathrm{a}$ & & $13.21 \mathrm{~d}$ & $15.43 c$ & $19.18 b$ & $20.52 \mathrm{a}$ & \\
\hline
\end{tabular}

Means designed by the same letter at each cell are not significantly different at the $5 \%$ level according to Duncan's multiple range tests. 


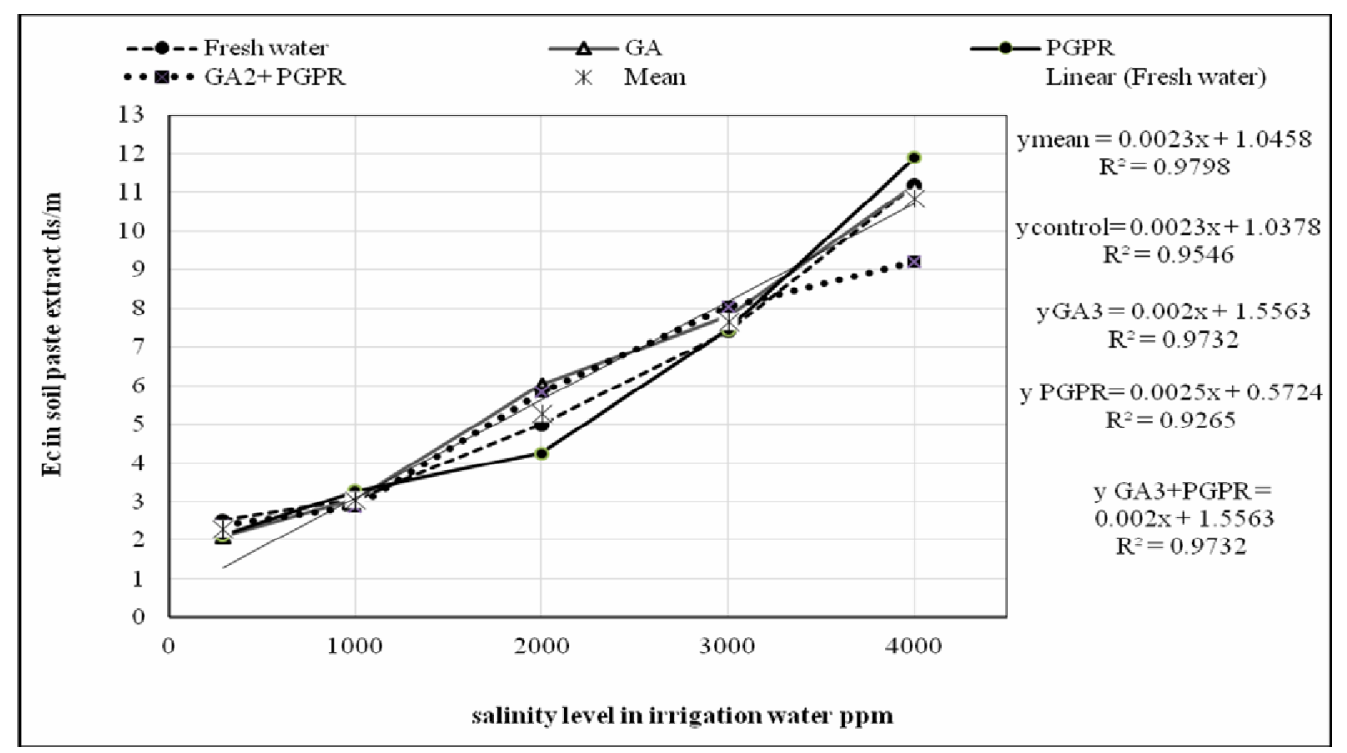

Figure.3. Correlation of Soil salinity at the end of experiment affected by saline irrigation water levels and foliar applications

\section{DISCUSSION}

The results reported in the present study displayed a general decline in the growth of Nigella sativa L. plants as far as plant height, the number of branches, plant dry weight, number of capsules, weight of capsules, 1000 seeds weight and seed yield (Tables 1, 2 and 3). The inhibition in growth parameters by salinity stress was previously reported by (Khan et al., 2010) on Linum usitatissimum L., (Bourgou et al., 2012) on Nigella sativa L. and (Saeidi-Sar et al., 2013) on Phaseolus vulgaris seedlings. Salinity can hamper plant growth by altering the water potential, increasing the ion toxicity, impeding the cell division besides cell expansion, or causing an ion imbalance (Arshi et al., 2005). In this perspective, (Younis et al., 2010) stated that the growth reduction initiated by salinity stress due to inhibiting apical growth in plants in addition to an endogenous hormonal imbalance. In both situations, the reduction could have been produced by the lethal effects of ions $\left(\mathrm{Na}^{+}\right.$and $\left.\mathrm{Cl}^{-}\right)$on metabolism or from adverse water relations.

In our study, the growth features of black cumin under salinity stress was effectively enhanced with $\mathrm{GA}_{3}$ supplement, however, the harmful influences of salinity was strong enough to hamper plant growth because of a decrease in gibberellin production. Therefore, the addition of gibberellic acid might increase seedling growth by enhancing endogenous gibberellin content as mentioned by (Rodr?guez et al., 2006). Furthermore, the improvement of growth rate by gibberellin might result in an enlargement of leaf area, the motivation of cell division and/or cell elongation, stimulation of photosynthetic rate, modified partitioning of photosynthates, or in their combination. The $\mathrm{GA}_{3}-$ mediated invertase activity in elongating shoots could result in an accumulation of hexoses which considered important for the primary cell wall biosynthesis, accordingly enhancing seedling growth beneath stress condition (Saeidi-Sar et al., 2007). Enhancing plant growth under salinity stress by $\mathrm{GA}_{3}$ has formerly been reported by (Ashraf et al., 2002, Khan et al., 2010) on wheat and Linum usitatissimum $\mathrm{L}$ plants.

Increasing levels of saline irrigation water produced a clear reduction in branches number, capsules number, dry weight of plants, seed yield, capsules yield and RWC of black cumin; nevertheless, the decline in the aforementioned organs was partially overcome by PGPR . The positive effect of PGPR on plant growth may be attributed to that PGPR motivation plant in growth and productivity via direct or indirect mechanisms. Direct mechanisms include plant hormone creation, improved iron accessibility, phosphorus solubilization and nutrient mobilization are a portion of the direct methods of growth development by PGPR . Indirect growth promotion happens when PGPR encourage plant growth by improving growth-restricting conditions.

Production of antagonistic materials to eliminate specific destructive microbes from roots vicinity and initiation of systemic resistance provides fortification against pathogens so improving growth-promoting conditions as reported by (Pierson and Thomashow, 1992 and Weller et al., 2002). Our results are in harmony with data from other researchers. They reported that the foliar treatment of PGPR had a considerable effect on alleviation salt stress, PGPR from medicinal plants such Withania somnifera, 
Catharanthus roseus, Coleus forskohlii, Ocimum sanctum and Aloe vera have been stated to increase growth and yield (Attia and Saad, 2001; Karthikeyan et al., 2008).

The decrease in Nigella Sativa L. root parameters ( Table 2 ) such weight decline as a result of salinity stress is formerly documented on Phaseolus vulgaris L., (Saeidi-Sar et al., 2013) on linseed (Khan et al., 2010)

Ashraf et al., (2002) reported that fresh and dry weights of roots, were decreased with increasing salt amount for Triticum aestivum L. Applying $\mathrm{GA}_{3}$ clearly improved black cumin root growth this confirms earlier reports on various plant species. Saeidi-Sar et al., (2013) found that common bean seedlings were less affected as a result of $\mathrm{GA}_{3}$ applications and almost exhibited no root growth reduction under salty conditions, but $\mathrm{GA}_{3}$ increased linseed root dry weight under salt stress (Khan et al., 2010). Moreover, the gibberellic acid treatment caused a significant effect on fresh and dry weight of both spring wheat cultivars (Ashraf et al., 2002). In addition applying PGPR generally resulted in an obvious increase in Nigella sativa L. root growth and the inhibitory impact of salinity stress was fully ameliorated particularly at low salinity level. Our findings are in harmony with many

authors who revealed that PGPR had a significant impact on alleviation of salt stress. Egamberdieva et al., (2013) stated that PGPR significantly improved root length, shoot length and total biomass of Silybum marianum (milk thistle ) plants subjected to salt stress after using Pseudomonas extremorientalis TSAU20 by producing auxin, exopolysaccharide, biofilm creation, as well as Saravanakumar and Samiyappan, (2007) found that applying PGPR increased salt tolerance of Arachis hypogaea through lowering ethylene production, auxin production, exopolysaccharide. On the same plant (Nautiyal et al., 2013) showed an increment in fresh biomass, total length and root length over control under salt stress by using PGPR through the manufacture of $\mathrm{NH}_{3}$, siderophore, chitinase, $\mathrm{HCN}$, IAA production and phosphorus solubilization.

As our data revealed, a decrease in chlorophyll content in relation to the undesirable effect of prolonged saline water stress (Table 4 ) which might be anticipated to a reduction in the uptake of minerals such as $\mathrm{Mg}$ and $\mathrm{N}$ required for chlorophyll biosynthesis or membrane deterioration (Sheng et al., 2008). In addition to the uncertainty of protein complexes and damage of chlorophyll by the raised activity of chlorophylldegrading enzyme chlorophyllase under stress circumstances (Reddy and Vora, 1986). Numerous reports proved that leaves total chlorophyll content was lessened by rising salinity level ( Tuna et al., 2008; Shoresh et al., 2011; Celik and Atak, 2012). The results also, indicated that PGPR treatment support greater chlorophyll concentration under saline situation and these findings were consistent with several authors who reported that PGPR increase chlorophyll content in mung bean plants (Dutta et al., 2005) and maize (Nadeem et al., 2007), (Nabti et al., 2010) reported that inoculation durum wheat (Triticum durum var. Waha) with the rhizosphere bacterium azospirillum brasilense under saline environments increased chlorophyll content. Moreover, the obtained results showed the beneficial effect of $\mathrm{GA}_{3}$ treatment on chlorophyll content under saline situations which is in agreement with (Misratia et al., 2013) who mentioned that $\mathrm{GA}_{3}$ increased photosynthetic capacity an essential feature for greater dry matter synthesis in rice salt-stressed plants. Also, applying $\mathrm{GA}_{3}$ increased chlorophyll levels for both spring wheat cultivars (Triticum aestivum L.) exposed to salinity circumstances (Ashraf et al., 2002). On chamomile plant, chlorophyll degradation occurred by salinity was prohibited by using $\mathrm{GA}_{3}$ (Ali and Hassan, 2014). Furthermore, spraying the vegetative parts of maize, wheat, cotton, broad and parsley plants with $\mathrm{GA}_{3}$ increased pigments content (Abd El-Samad and Shaddad, 2014). This because the role of $\mathrm{GA}_{3}$ for the inhibition of pigment degradation or motivation of protochlorophyllide synthesis by phytohormons (Pazuki et al., 2013) and this may be a vital part of a defense versus salinity stress.

Our results display that salinity stress induces membrane permeability changes (Table 4) which are in agreement with results achieved by $\mathrm{NaCl}$ application (Ali and Hassan, 2014) on chamomile plant. Additionally, when salt-stressed maize inoculated with PGPR, ACC deaminase comprising Pseudomonas syringae, Enterobacter aerogenes and P. fluorescens caused high relative water content (Nadeem et al., 2007) . Highest leaf (RWC) and least (MP) have been certified in wheat and barley treated with PGPR strains of Bacillus and Azospirillum (Turan et al., 2012). Greater cell wall flexibility and the capability to modify plant hormones are particular mechanisms induced by Azospirillum to combat with salinity and osmotic stress ( Creus et al., 1998 and Bashan et al., 2004 ). On the other hand, $\mathrm{GA}_{3}$ counteracts with salinity stress by rising membrane permeability and nutrient amounts in leaves which finally leads to superior seedling growth, shoot, root and whole biomass (Iqbal et al., 2012). Also, (Ali and Hassan, 2014) found that membrane stability index for chamomile plants was prevented when salinity treatments were combined with $\mathrm{GA}_{3}$.

Proline accumulation in plant tissues is a valuable physiological reaction to counterbalance saline stress. 
Proline performs a defensive function against salinity disorders in plants (Verbruggen and Hermans, 2008). The significantly improved proline levels located in Nigella Sativa L. plants during harsh salt stress (Table 5) reflect this response. Such proline accumulation in consequence of salt stress is well documented ( Nabti et al., 2010; Ali and Hassan, 2014 and Shao et al., 2015). As our data indicated, salinity mitigation by $\mathrm{GA}_{3}$ may occur through its effect on proline metabolism via regulating $\mathrm{N}$ accumulation (Iqbal and Ashraf, 2013). In addition (Tuna et al., 2008) reported that foliar treatment of $\mathrm{GA}_{3}$ improved proline content which lessened antagonistic impacts of salinity by maintaining membrane permeability, increasing macro and micronutrient levels. This superior gathering of proline could characterize a major biochemical adaptation in plants osmotic adjustment (Siddiqui et al., 2008).

An increment in CAT and POX enzymes activities we noticed with rising salinity levels (Table 5). Also, a secondary aspect of salinity in plants is the stressinduced creation of reactive oxygen species (ROS) (Manchanda and Garg, 2008). The enriched production of (ROS) through salinity stress lead to the advanced oxidative damage and finally cell death and growth suppression (Ruiz-Lozano et al., 2012). Thus, to keep metabolic tasks under stress, the scavenging of ROS is required. ROS scavenging depends on detoxification method offered by antioxidant enzymes (CATand POX).Under salt stress, plants displayed the enhanced amount of enzymes activities (CAT and POX), contrasted with their control. $\mathrm{GA}_{3}$ may likewise enhance salinity tolerance by keeping up enzyme activities. It is, accordingly, possible that foliar utilization of $\mathrm{GA}_{3}$ could be a helpful tool in supporting great seedling growth and establishment under salty conditions. Moreover, exogenous treatment with growth hormones may possibly be beneficial to return metabolic activities toward their regular levels (Iqbal et al., 2012). Also, (Tuna et al., 2008) stated a similar impact of $\mathrm{GA}_{3}$ on the antioxidant levels .The findings showed that foliar treatment of $\mathrm{GA}_{3}$ was observed to be efficient in lightening the unfavorable impact of salt stress by improving antioxidants activity which is consistent with (Ali and Hassan, 2014) on chamomile and (Saeidi-Sar et al., 2013) on Phaseolus vulgaris L. seedlings. PGPR is also reported to protect the plants from saline disorders by decreasing membrane destabilizing activity in the cell (Khan and Panda, 2008). Moreover, PGPR improves ROS-scavenging enzymes such as catalase and ascorbate peroxidase (Kohler et al.,2010 and Gururani et al., 2013).

The increase in total soluble salts (Figure 3) may be proportional to the salts from saline irrigation water these results agree with those obtained by
(Mostafazadeh-fard et al., 2007 and Noufal et al., 2008). However, utilizing freshwater and $1000 \mathrm{ppm}$ sea water decreased soil EC by 39 and $18 \%$, respectively compared to soil EC before planting this anticipated by addition of leaching requirements ( Mostafazadeh-fard et al., 2007 and Mostafazadeh-Fard et al., 2008). The values of EC differ between spraying treatments; the greatest values of soil EC were recorded after using $\mathrm{GA}_{3}$ spraying treatments.

As a conclusion, salinity treatments negatively influenced the growth and yield characters of Nigella sativa plants. Under salinity treatments relative water content, chlorophyll content and fixed oil percentage were decreased. However, proline, membrane permeability, enzyme activities and total soil salts were increased. Meanwhile, $\mathrm{GA}_{3}$ or PGPR treatments lightened the harmful impacts of salinity on the formerly declared parameters. $\mathrm{GA}_{3}$ or PGPR treatment increased proline content and activities of CAT and POX which may consider promising mechanisms for salinity alleviation in Nigella sativa $\mathrm{L}$. plant.

\section{ACKNOWLEDGEMENT}

The authors are so grateful to Microbiology Department, Sakha Agricultural Research Station, Agricultural Research Center for offering the microbial cultures needed for the experiment.

\section{REFERENCES}

Abd El-Samad, H. M.A and M.A.K Shaddad. 2014. The exogenous amelioration roles of growth regulators on crop plants grown under different osmotic potential. J. Stress Physiol. Biochem. 10:203-213.

Ali,E.F.and F.A.S. Hassan.2014. Alleviatory effects of salt stress by mycorrhizal fungi and gibberellic acid on chamomile plant. Int. J. Scinece Res. 3:109-118.

Arshi,A., M.Z.Abdin and M.Iqbal. 2005 . Ameliorative effects of $\mathrm{CaCl} 2$ on growth, ionic relations, and proline content of Senna under salinity stress. J. Plant Nutr. 28:101-125.

Ashraf, M., F.Karim and E. Rasul. 2002. Interactive effects of gibberellic acid (GA3) and salt stress on growth, ion accumulation and photosynthetic capacity of two spring wheat (Triticum aestivum L.) cultivars differing in salt tolerance. Plant Growth Regul. 36:49-59.

Attia, F. A. and O. A. O. Saad. 2001. Biofertilizers as potential alternative of chemical fertilizer for Catharanthus roseus G. Don. J. Agric. Sci. 26:7193-7208.

Baghalian, K., A.Haghiry, M. R.Naghavi and A. Mohammadi. 2008. Effect of saline irrigation water on agronomical and phytochemical characters of chamomile (Matricaria recutita L .). Sci. Hortic. (Amsterdam). 116:437-441.

Bashan, Y. , J.P. Hernandez, L .A. Leyva, M. Bacilio. 2002. Alginate microbeads as inoculant carriers for plant growth-promoting bacteria. Biol. Fertil. Soils.35:359-368.

Bashan, Y., G.Holguin and L. E. De-Bashan. 2004. Azospirillum-plant relationships: physiological, 
molecular, agricultural, and environmental advances 1997-2003. Can. J. Microbiol. 50:521-577.

Bates, L. S., R. P.Waldren and I. D. Teare. 1973. Rapid determination of free proline for water-stress studies. Plant Soil. 39:205-207.

Bohm, W . 2012. Methods of studying root systems. Springer Science \& Business Media.

Bourgou, S., I. Bettaieb, I. Hamrouni and B. Marzouk. 2012. Effect of $\mathrm{NaCl}$ on fatty acids, phenolics and antioxidant activity of Nigella sativa organs. Acta Physiol. Plant.34: 379-386

Bourgou, S., R. Ksouri, A. Bellila, I. Skandrani, H.Falleh and B. Marzouk. 2008. Phenolic composition and biological activities of Tunisian Nigella sativa L. shoots and roots. C. R. Biol. 331:48-55.

Bourgou, S., A.Pichette, B. Marzouk and J. Legault. 2010. Bioactivities of black cumin essential oil and its main terpenes from Tunisia. South African J. Bot. 76:210-216.

Bradford, M.M.1976. A rapid and sensitive method for the quantitation of microgram quantities of protein utilizing the principle of protein-dye binding. Anal. Biochem. $72: 248-254$.

Celik, O. and C. Atak. 2012. The effect of salt stress on antioxidative enzymes and proline content of two Turkish tobacco varieties. Turkish J. Biol. 36:339-356.

Cheikh-Rouhou, S., S.,Besbes, B. Hentati, C.Blecker, C.Deroanne and H. Attia.2007. Nigella sativa L.: Chemical composition and physicochemical characteristics of lipid fraction. Food Chem. 101:673-681.

Claiborne, A. L. 1985. Catalase activity, in: Handbook of Methods for Oxygen Radical Research. CRC Press Boca Raton. pp. 283-284.

Creus, C. M., R. J.Sueldo and C .A. Barassi. 1998. Water relations in Azospirillum-inoculated wheat seedlings under osmotic stress. Can. J. Bot. 76:238-244.

Dutta,S., R. P.Singh and J .K. Jindal. 2005. Effect of antagonistic bacteria and plant defence activators on management of bacterial leaf spot of mungbean. Indian Phytopathol. 58:269-275.

Egamberdieva, D., D. Jabborova and N. Mamadalieva. 2013. Salt tolerant Pseudomonas extremorientalis able to stimulate growth of Silybum marianum under salt stress. Med. Aromat. Plant Sci. Biotechnol. 7:7-10.

Flowers, T. J., A.Garcia, M.Koyama and A .R. Yeo. 1997. Breeding for salt tolerance in crop plants the role of molecular biology. Acta Physiol. Plant. 19:427-433.

Ghoulam, C., A.Foursy and K. Fares. 2002. Effects of salt stress on growth, inorganic ions and proline accumulation in relation to osmotic adjustment in five sugar beet cultivars. Environ. Exp. Bot. 47:39-50.

Gururani, M .A., C. P.Upadhyaya, V. Baskar, J.Venkatesh, A. Nookaraju and S. W. Park. 2013. Plant growth-promoting rhizobacteria enhance abiotic stress tolerance in Solanum tuberosum through inducing changes in the expression of ROS-scavenging enzymes and improved photosynthetic performance. J. Plant Growth Regul. 32:245-258.
Hassan, F. A. S. and S. A. Mahfouz. 2012. Effect of 1methylcyclopropene (1-MCP) on the postharvest senescence of coriander leaves during storage and its relation to antioxidant enzyme activity. Sci. Hortic. (Amsterdam). 141:69-75.

Hoque, M. M. and I .S. Haque. 2002. Yield Parameters of mungbean (Vigna radiata L.). Pakistan J. Biol. Sci. 5:281283.

Horwitz, W., P. Chichilo and H. Reynolds . 1970. Official methods of analysis of the Association of Official Analytical Chemists. Washington, DC, USA

Iqbal, M. and M. Ashraf. 2013. Gibberellic acid mediated induction of salt tolerance in wheat plants: Growth, ionic partitioning, photosynthesis, yield and hormonal homeostasis. Environ. Exp. Bot. 86:76-85.

Iqbal, N., A. Masood and N .A. Khan. 2012. Phytohormones in Salinity Tolerance: Ethylene and Gibberellins Cross Talk., in: Khan, N.A., Nazar, R., Iqbal, N., Anjum, N.A. (editors.), Phytohormones and Abiotic Stress Tolerance in Plants. Springer-Verlag Berlin Heidelberg.pp. 77-98.

Jensen, H. L. 1951. Notes on the biology of Azotobacter, in: Proceedings of the Society for Applied Bacteriology. pp. 89-94.

Karthikeyan, B., C. A. Jaleel, G. M .A.Lakshmanan and M. Deiveekasundaram. 2008. Studies on rhizosphere microbial diversity of some commercially important medicinal plants. Colloids surfaces B Biointerfaces. 62:143-145.

Khan, M. H. and S.K, Panda. 2008. Alterations in root lipid peroxidation and antioxidative responses in two rice cultivars under $\mathrm{NaCl}$-salinity stress. Acta Physiol. Plant. 30:81-89.

Khan, M .N., M. H.Siddiqui and F.Mohammad, M.Naeem, M. M .A. Khan.2010. Calcium chloride and gibberellic acid protect linseed (Linum usitatissimum $L$.) from $\mathrm{NaCl}$ stress by inducing antioxidative defence system and osmoprotectant accumulation. Acta Physiol. Plant. 32:121-132.

Kohler, J., F. Caravaca and A. Rold?n. 2010. An AM fungus and a PGPR intensify the adverse effects of salinity on the stability of rhizosphere soil aggregates of Lactuca sativa. Soil Biol. Biochem. 42:429-434.

Maggio, A., G.Barbieri, G.Raimondi and S. De Pascale. 2010. Contrasting effects of GA3 treatments on tomato plants exposed to increasing salinity. J. Plant Growth Regul. 29:63-72.

Manchanda, G. and N. Garg. 2008. Salinity and its effects on the functional biology of legumes. Acta Physiol. Plant. 30:595-618.

Matthaus, B. and M .M. Ozcan. 2011. Fatty Acids, Tocopherol, and Sterol Contents of Some Nigella Species Seed Oil. Czech J. Food Sci. 29:145-150.

Misratia, K. M., M .R.Ismail, M. A.Hakim, M .H. Musa and A. Puteh. 2013. Effect of salinity and alleviating role of gibberellic acid (GA3) for improving the morphological, physiological and yield traits of rice varieties. Aust. J. 
Crop Sci. 7:1682-1692.

Moran, R. 1982. Formulae for determination of chlorophyllous pigments extracted with $\mathrm{N}, \mathrm{N}-$ dimethylformamide. Plant Physiol. 69:1376-1381.

Mostafazadeh-fard, B., M.Heidarpour, Q. A.Aghakhani and M. Feizi. 2007. Effects of irrigation water salinity and leaching on soil chemical properties in an arid region. Int. J. Agric. Biol. 3:166-469.

Mostafazadeh-Fard, B., M.Heidarpour, Q.A.Aghakhani and M. Feizi. 2008. Effects of leaching on soil desalinization for wheat crop in an arid region. Plant Soil Environ. 54:20-29.

Nabti, E., M.Sahnoune, M.Ghoul, D.Fischer, A. Hofmann, M. Rothballer, M. Schmid and A. Hartmann.2010. Restoration of Growth of Durum Wheat (Triticum durum var. waha) Under Saline Conditions Due to Inoculation with the Rhizosphere Bacterium Azospirillum brasilense $\mathrm{NH}$ and Extracts of the Marine Alga Ulva lactuca. J. Plant Growth Regul. 29:6-22.

Nadeem, S .M., Z .A.Zahir, M.Naveed and M. Arshad. 2007. Preliminary investigations on inducing salt tolerance in maize through inoculation with rhizobacteria containing ACC deaminase activity. Can. J. Microbiol. 53:11411149.

Nautiyal, J., M.Christian and M .G. Parker. 2013. Distinct functions for RIP140 in development, inflammation, and metabolism. Trends Endocrinol. Metab. 24:451-459.

Noufal, E., O. H.El-Hussieny and I. M .Farid.2008. Effect of irrigation with increasing water salinity levels using seafresh mixed water on some soil chemocal properties and plant growth, in: The 3rd International Scientific Conference for Environment. South Valley University, Egypt,November. pp. 80-94.

Oueslati, S., K.bouraoui, N. H. da A., M.Rabhi, R.Ksouri and M.Lachaal. 2010. Physiological and antioxidant responses of Mentha pulegium (Pennyroyal ) to salt stress. Acta Physiol. Plant. 32:289-296.

Page, A. L., R .H.Miller and D. R.Keeney. 1982. Methods of Soil Analysis: Chemical and Microbiological proerpteis. Madison, Wisconsin.

Pazuki, A., M. Sedghi and F. Aflaki. 2013. Interaction of salinity and phytohormones on wheat photosynthetic traits and membrane stability. Agriculture. 59:33-41.

Pierson, L. S. and L .S. Thomashow. 1992. Cloning and heterologous expression of the phenazine biosynthetic locus from Pseudomonas aureofaciens 30-84. Mol. PlantMicrobe Interact. 5:330-339.

Rajasekaran, L. R. and T. J. Blake. 1999. New plant growth regulators protect photosynthesis and enhance growth under drought of jack pine seedlings. J. Plant Growth Regul. 18:175-181.

Ramadan, M. F. 2007. Nutritional value, functional properties and nutraceutical applications of black cumin (Nigella sativa L.): an overview. Int. J. food Sci. Technol. 42:1208-1218.
Reddy, M. P. and A. B. Vora.1986. Salinity induced changes in pigment composition and chlorophyllase activity in wheat. Indian J. Plant Physiol. 29:331-334.

Rodr?guez, A. A., A. M.Stella, M .M.Storni, G.Zulpa and M.C. Zaccaro.2006. Effects of cyanobacterial extracellular products and gibberellic acid on salinity tolerance in Oryza sativa L. Saline Systems 2:2-7.

Ruiz-Lozano, J .M., R.Porcel, C.Azc?n and R. Aroca.2012 . Regulation by Arbuscular mycorrhizae of the integrated physiological response to salinity in plants: new challenges in physiological and molecular studies. J. Exp. Bot. 63:4033-4044.

Saeidi-Sar, S., H.Abbaspour, H.Afshari and S .R Yaghoobi. 2013. Effects of ascorbic acid and gibberellin $\mathrm{GA}_{3}$ on alleviation of salt stress in common bean (Phaseolus vulgaris L.) seedlings. Acta Physiol. Plant. 35:667-677.

Saeidi-Sar ,S., R. A.Khavari-Nejad, H.Fahimi, M. Ghorbanli and A.Majd. 2007. Interactive effects of gibberellin GA3 and ascorbic acid on lipid peroxidation and antioxidant enzyme activities in Glycine max seedlings under nickel stress. Russ. J. Plant Physiol. 54:74-79.

Sakamoto, A. and N. Murata. 2002. The role of glycine betaine in the protection of plants from stress: Clues from transgenic plants. Plant. Cell Environ. 25:163-171.

Saravanakumar, D. and R .Samiyappan. 2007. ACC deaminase from Pseudomonas fluorescens mediated saline resistance in groundnut (Arachis hypogea) plants. J. Appl. Microbiol. 102:1283-1292.

Shannon, L. M., E.Kay and J .Y. Lew. 1966. Peroxidase isozymes from horseradish roots I. Isolation and physical properties. J. Biol. Chem. 241:2166-2172.

Shao,Y., J.Gao, X. Wu, Q.Li, J.Wang, P.Ding and X.Lai. 2015. Effect of salt treatment on growth, isoenzymes and metabolites of Andrographis paniculata ( Burm . f .) Nees. Acta Physiol. Plant. 37:1-12

Sheng, M., M. Tang, H.Chen, B. Yang, F. Zhang and Y. Huang.2008. Influence of arbuscular mycorrhizae on photosynthesis and water status of maize plants under salt stress. Mycorrhiza. 18:287-296.

Shoresh, M., M.Spivak and N. Bernstein.2011. Involvement of calcium-mediated effects on ROS metabolism in the regulation of growth improvement under salinity. Free Radic. Biol. Med. 51:1221-1234.

Siddiqui, M .H., M. N.Khan, F. Mohammad and M. M .A. Khan.2008. Role of nitrogen and gibberellin (GA3) in the regulation of enzyme activities and in osmoprotectant accumulation in Brassica juncea L. under salt stress. J. Agron. Crop Sci. 194:214-224.

Snedecor, G. W. and W .G. Cochran.1980. Statistical Methods, Seventh Edition (Ames, IA: The Iowa State University Press), Seventh Ed. ed. Iowa, USA.

Steel, R. G. D., J. H.Torrie and D. A. Dickey. 1980. Principles and Procedures of Statistic: A Biometrical approach. 2nd Ed. McGraw-Hill Publ. Co., New York. 631p.

Tuna, A. L., C. Kaya, M.Dikilitas and D. Higgs. 2008. The 
combined effects of gibberellic acid and salinity on some antioxidant enzyme activities, plant growth parameters and nutritional status in maize plants. Environ. Exp. Bot. 62:1-9.

Turan, M., M. Gulluce and F. Sahin. 2012. Effects of plantgrowth-promoting rhizobacteria on yield, growth, and some physiological characteristics of wheat and barley plants. Commun. Soil Sci. Plant Anal. 43:1658-1673.

Verbruggen, N. and C. Hermans. 2008. Proline accumulation in plants: A review. Amino Acids. 35:753-759.

Weller, D. M., J .M.Raaijmakers, B. B. M.Gardener and L .S. Thomashow. 2002. Microbial populations responsible for specific soil suppressiveness to plant pathogens. Annu. Rev. Phytopathol. 40:309-348.

Weyens, N., D. van der Lelie, S. Taghavi, L.Newman and J. Vangronsveld. 2009. Exploiting plant--microbe partnerships to improve biomass production and remediation. Trends Biotechnol. 27:591-598.

Whetherley, P. E. 1950. Studies in the water relations of cotton plants. I. The field measurement of water deficit in leaves. New Phytol. 49:81-97.

Yamaguchi, S. 2008. Gibberellin metabolism and its regulation. Annu. Rev. Plant Biol.59: 225-251

Yan, B., Q.Dai, X.Liu, S.Huang and Z.Wang. 1996. Floodinginduced membrane damage, lipid oxidation and activated oxygen generation in corn leaves. Plant Soil. 179:261268.

Yang, J., J. W. Kloepper and C.M. Ryu. 2009. Rhizosphere bacteria help plants tolerate abiotic stress. Trends Plant Sci. 14:1-4.

Younis, M. E., M. N. A.Hasaneen and A. M. S. Kazamel. 2010. Exogenously applied ascorbic acid ameliorates detrimental effects of $\mathrm{NaCl}$ and mannitol stress in Vicia faba seedlings. Protoplasma. 239:39-48.

Zhu, J.K .2002. Salt and drought stress signal transduction in plants. Annu. Rev. Plant Biol. 53:247-273.

\section{الملخص العربي}

\section{تخفيف الاجهاد الملحى لحبة البركه بالجبريللين والريزوبكتريا \\ ناهد مصطفى راثد، عوض يوسف شعله، محمود عبداله محمود}

الكبسولات وعدد الجذور لكل نبــات و الــوزن الطــازج

و الجاف للجذور ومحصول الكبـسولات و البـذور ووزن . . . . بذره بالمقارنه بالكنترول كما أدت الملوحه لتقليـل تزكيز الكلوروفيل و النسبه المئويه للزيت الثابت و المحتــىى النسبى للماء ومع ذلك ازداد محتوى البرولين ونشاط انزيم البيروكسيديز و الكتاليز ونفاذية الغشاء و المحتــوى الكلـى دئى

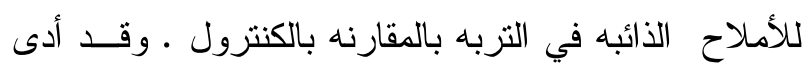

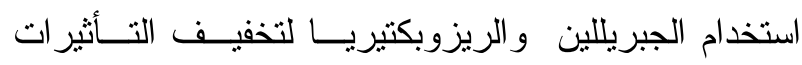
الضـاره للملوحه. الزياده في نــشاط الانزيمــات وتــر اكم البرولين يرجع الى الجبريللين و الريزوبكثريا و الذى اقترح

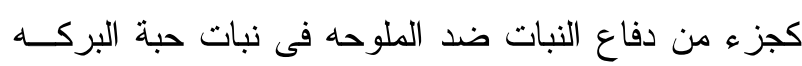

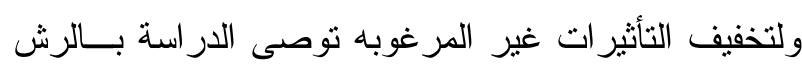
بالجبريللين بتركيز . 1 جزء في المليون أو الريزوبكيتزيــا

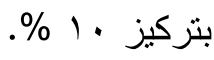

تعتبر الملوحة احد اهم الاجهادات الخطيرة للنباتات مما يؤثر على العمليات الاخرى مثل الاجهادات التاكسدية والتى

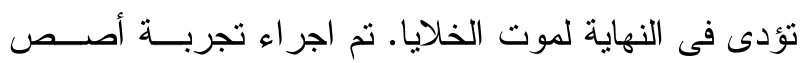

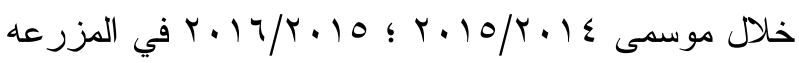
التجريبيه بمحطة بحوث البساتين بسخا لدراسة امكانية تقليل

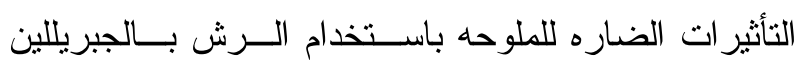

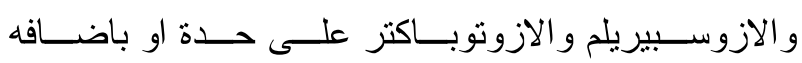
الجبريللين و الازوسبيريلم و الازوتوباكتر معا علـىى النــــو الخضرى و المحصول و التركيب الكيماوى و النسبه المئويـــهـ للزيت الثابت لنبات حبة البركه وكانت معاملات الملوحــه عباره عن ماء عادى كنترول وماء بحر مخفف الى ...

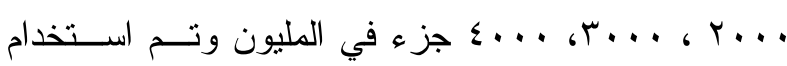

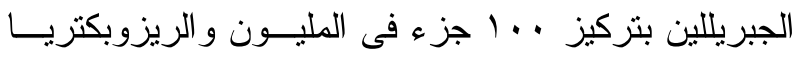
بتركيز • 1 \% وقد أدت معاملات الملوحه الى تقليل طــــ النبات و وعدد الأفرع و الــوزن الجــاف للنبــات و وعـدد 